\title{
An Improved Parallel Compact Scheme for Domain- Decoupled Simulation of Turbulence
}

\author{
J. Fang ${ }^{1}$, F. Gao ${ }^{2}$, C. Moulinec ${ }^{1}$ and D. R. Emerson ${ }^{1}$ \\ ${ }^{1}$ Scientific Computing Department, STFC Daresbury Laboratory, Warrington WA4 4AD, \\ United Kingdom \\ ${ }^{2}$ Faculty of Engineering and Physical Sciences, University of Surrey, Guildford GU2 7XH, \\ United Kingdom
}

\begin{abstract}
An improved interface-coupling compact scheme for first and second spatial derivatives is proposed for domain-decomposition-based parallel computational fluid dynamics. The method improves the accuracy of previously developed decoupled schemes and preserves the accuracy and bandwidth properties of fully-coupled compact schemes, even for a very large degree of parallelism, and enables the Navier-Stokes equations to be solved independently on each processor. The scheme is analysed using Fourier analysis and error analysis, and tested on one-dimensional wave-packet propagation, a two-dimensional vortex convection problem, and in the direct numerical simulation of the 3-D Taylor-Green vortex problem and turbulent channel flow. Our results demonstrate the scheme's effectiveness in performing direct numerical simulation of turbulence in terms of accuracy and scalability.
\end{abstract}

Keywords: High-order compact scheme; Finite difference method; Domain-decomposition; Large-scale parallel computation; Direct numerical simulation

\section{Introduction}

Compact or Padé-type schemes have been developed and studied for over 6 decades. In 1955, Kopal [1] presented a successive extrapolation method to solve differential equations, in which the conception of compact schemes was adopted to approximate first and second derivatives. Kopal mentioned that it represented the most accurate recursion formula employing three successive ordinates. In the 1970s, compact schemes for the finite difference method were further developed by Kreiss and Oliger [2,3], Adam [4,5] and Hirsh [6], among whom Hirsh [6] may be the first person to use a compact scheme for computational fluid dynamics (CFD) problems. They all have proved compact schemes are 
efficient and accurate in solving ordinary differential and partial differential equations. Lele [7] then presented a detailed analysis of compact schemes upto fourth derivatives, including their boundary closures. The small stencil and spectral-like resolution of compact schemes are highly desirable in CFD methods with structured meshes [8], especially for direct numerical $[9,10,11,12] /$ large-eddy simulation $[13,14]$ (DNS/LES) of turbulence and computational aeroacoustics (CAA) $[15,16,17,18]$, in which small-scale flow structures need to be resolved. Compact schemes have advantages regarding high accuracy, small truncation error, low dissipation, and a compact stencil. The penalty is the implicit nature of compact schemes, which means the solution of a linear narrow-banded system (e.g. a tridiagonal or pentadiagonal linear system) of equations for unknown derivative values is required. In a serial environment, a version of Gaussian elimination known as the Thomas algorithm can be used to solve a linear system having $N$ unknowns with $O(N)$ operations, but difficulties arise in a domain-decomposition-based parallel environment. In both forward and backward sweeps of the Thomas algorithm, the solution is recursive, and a local node depends on solutions from its neighbouring nodes.

Significant effort has been devoted to the parallelisation of compact schemes. In general, there are two categories of methods for dealing with a parallel compact scheme. The first group focuses on the method of solving a linear system in parallel, using either pipeline methods [19,20], alternating direction methods [21], parallel diagonal dominant algorithms $[22,23]$, or line-relaxation methods [24]. The second group essentially decouples compact schemes to enable them to be solved independently on each processor. Consequently, the computational field can be partitioned with classic domain decomposition methods [25] and each subdomain is solved independently on a processor, in common with most CFD solvers using explicit schemes. The term, decoupled, here means the operation of numerical differentiation inside a subdomain or processor is independent of other parts of the domain at a particular computational step. Physically, all parts of the domain are still coupled dependent on the problem being solved. The decoupled approach has shown superior parallel performance as demonstrated by Sengupta et al. [26], Chao et al. [27], Kim and Sandberg [28], and Kim [29]. The decoupling methods inevitably introduce interdomain interfaces, on which a boundary method is required to close the compact scheme inside of each subdomain. From this point of view, the decoupled scheme is similar to the concept of hybrid methods, where different schemes are applied in different regions of the domain, such as hybrid compact-WENO schemes [27, 30,31] and hybrid compact-TVD schemes [32]. The interdomain interface problem is also involved in multi-block solvers, in which the focus is mainly on the stability and conservation properties of the interface treatment methods [33, 34, 35, 36]. Carpenter et al. [33] derived stable and conservative interface 
conditions for high-order schemes for the scalar advection-diffusion equation on multiple domains. Nordström et al. [34, 35] further developed the method for the Navier-Stokes equations. Gao [37] proposed an interface flux-reconstruction method based on a Riemann

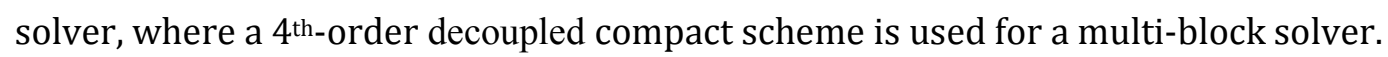

Different boundary methods for interdomain interfaces have been developed. A straightforward way is to use an explicit scheme at an interface to provide a boundary value to two adjacent subdomains. Chao et al. [27] first introduced the idea in developing a $5^{\text {th- }}$ order hybrid compact-WENO scheme. For the convective fluxes, an explicit $5^{\text {th-order }}$ upwind scheme was used at the interface to decouple a $5^{\text {th }}$-order compact upwind scheme, hybridising with a WENO scheme to capture shock-waves. For the computation of the diffusive fluxes, a $6^{\text {th }}$-order explicit central scheme was used at the block interface to decouple the $6^{\text {th }}$-order compact central scheme. Their decoupling method has shown good stability and scalability [27]. The same strategy was also adopted by Fico et al. [32]. Chao's method has a clear advantage that two adjacent subdomains are assigned the same interface value and no extra data exchange is needed, which helps to ensure good parallel efficiency. The drawback is that the benefits of the compact scheme, in terms of accuracy and error, might be lost at an interface. As noted by Chao et al. [27], the global resolution property is affected by the degree of parallelism.

Sengupta et al. [26] proposed a different approach for interdomain interfaces. They adopted biased boundary schemes and large node-overlapping to close and decouple the compact scheme in each subdomain. High-frequency spurious wave packets were observed due to the asymmetry of the biased boundary scheme propagating downstream. It was proposed that spurious wave packets could be removed by larger overlapping, or applying a compact low-pass filter, and the scheme was successfully applied in DNS of compressible flows.

Kim [38] proposed an optimised boundary compact scheme, in which a particular spline function was devised to extrapolate the objective function and its first derivative beyond boundaries. Kim and Sandberg [28] then developed the idea to decouple the compact scheme for parallel computations. They extended Kim's boundary formulation to halo nodes from the adjacent subdomains and the approximation of the derivatives at nodes close to the interface are then obtained to close the pentadiagonal matrix system within each subdomain. Furthermore, Kim and Sandberg [28] proposed a decoupled $6^{\text {th }}$-order compact filter with a prediction-correction procedure. They proved the developed compact scheme and compact filter provide linear stability and $4^{\text {th }}$-order accuracy throughout the domain. However, the scheme of Kim and Sandberg [28] presents non-uniformity in resolution characteristics across subdomain interfaces, and numerical dissipation arises accordingly. 
This drawback was then largely reduced by Kim [29] by implementing a linear-algebraic transformation technique to obtain quasi-disjoint pentadiagonal matrix systems. Kim's scheme was then successfully applied in simulations of acoustics problems [39, 40,41].

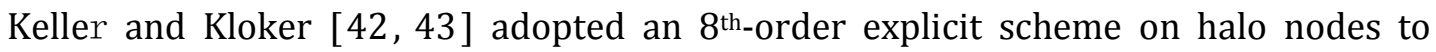
decouple the $6^{\text {th }}$-order compact scheme, and it is shown that the decoupled compact scheme has good robustness and accuracy. A linear speedup has been shown up to 4800 cores, and the computing time was less than the fully explicit scheme due to cache effects. They also discussed the effect of the number of ghost nodes, and eventually adopted one level of ghost nodes in DNS of boundary layer flows $[44,45,46]$. Recently, a similar approach was adopted by Capuano et al. [47] in developing a parallel compact finite-volume scheme, who used a $4^{\text {th-order }}$ explicit scheme and cell overlapping to decouple the compact scheme. Conservation is enforced by another application of the compact formula around interdomain interfaces. Their result has shown the conservation property of the finitevolume method is retained, and the accuracy is also well preserved within an acceptable tolerance.

Inspired by Sengupta et al. [26], Chao et al. [27], Kim [28], Keller and Kloker [42,43], and Capuano et al. [47], this paper presents a modified class of decoupled finite difference

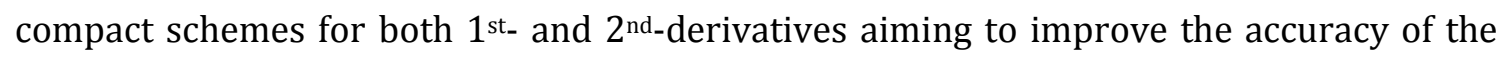
decoupled compact scheme. An explicit scheme, with the same order of accuracy as the corresponding compact scheme, is employed on the halo nodes to close the tridiagonal system within each subdomain, and a correction step is then introduced to further improve the accuracy of the scheme. The analysis and tests demonstrate that the proposed scheme has bandwidth properties and truncation errors close to the fully coupled scheme. Scalability is ensured because the scheme is decoupled at the interface of each subdomain. The scheme is assessed for the one-dimensional (1-D) linear equation for the wave-packet propagation problem and the two-dimensional (2-D) Euler equations for the vortex convection problem. It is then used to solve the three-dimensional (3-D) Navier-Stokes equations for DNS of the Taylor-Green Vortex problem and turbulent channel flow demonstrating its effectiveness in DNS of turbulent flows as well as its good scalability.

\section{Development of a domain-decoupled compact scheme}

In this section, the principle of the proposed decoupled compact (DCOM) scheme for

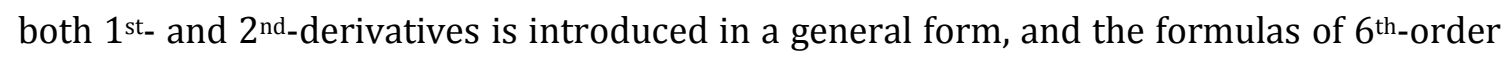
DCOM central schemes are also given in detail.

\subsection{First-derivative}

For a general continuously differentiable function, $\phi$, discretised on a uniform 1-D mesh 
$x_{i}=i \Delta, i=0,1, \cdots, N$, in which $\Delta$ is the distance between two consecutive mesh nodes. The derivative, $\phi^{\prime}$, at the $\mathrm{i}^{\text {-th }}$ mesh node (internal node) is approximated using the following general compact central scheme,

$$
\alpha \tilde{\phi}_{i-1}^{\prime}+\tilde{\phi}_{i}^{\prime}+\alpha \tilde{\phi}_{i+1}^{\prime}=\frac{1}{\Delta} \sum_{m=1}^{S} a_{m}\left(\phi_{i+m}-\phi_{i-m}\right),
$$

where $\tilde{\phi}_{i}^{\prime}$ is the approximation of $\phi^{\prime}$ at $x_{i}$, and $\alpha$ and $a_{m}$ are coefficients defining a specific scheme with a $2 S$ wide stencil. For nodes close to a physical boundary, modified schemes are needed to ensure the scheme's stencil does not go beyond the boundary, whose general formulas are given as $[8,48]$,

$$
\tilde{\phi}_{0}^{\prime}+\alpha_{0} \tilde{\phi}_{1}^{\prime}=\frac{1}{\Delta} \sum_{m=0}^{s_{0}} b_{m} \phi_{m}
$$

and

$$
\alpha_{1} \tilde{\phi}_{0}^{\prime}+\tilde{\phi}_{1}^{\prime}+\alpha_{1} \tilde{\phi}_{2}^{\prime}=\frac{1}{\Delta} \sum_{m=0}^{S_{1}} c_{m} \phi_{m}
$$

for the node on the boundary ( $i=0)$ and the first node away from the boundary $(i=1)$, respectively, and the coefficients, $\alpha_{0}, b_{m}, \alpha_{1}$ and $c_{m}$, define specific schemes. The widths of the stencils are given by $S_{0}$ and $S_{1}$, respectively. Symmetric operations can be employed for the $i=N$ and $i=N-1$ nodes.

Although compact schemes have the advantage of a smaller stencil, higher bandwidth resolution, and smaller truncation errors over an explicit scheme of the same order, the drawback is the implicit solution of Eq. (1-3). The following linear algebra system needs to be solved to obtain $\tilde{\phi}^{\prime}{ }_{i}$,

$$
\left[\begin{array}{cccccccc}
1 & \alpha_{0} & & & & & \\
\alpha_{1} & 1 & \alpha_{1} & & & & \\
& \alpha & 1 & \alpha & & & \\
& & & \ddots & & & \\
& & \alpha & 1 & \alpha & \\
& & & \alpha_{1} & 1 & \alpha_{1} \\
& & & & \alpha_{0} & 1
\end{array}\right]\left[\begin{array}{c}
\tilde{\phi}^{\prime}{ }_{0} \\
\tilde{\phi}_{1}^{\prime} \\
\tilde{\phi}^{\prime}{ }_{2} \\
\vdots \\
\tilde{\phi}^{\prime}{ }_{N-2} \\
\tilde{\phi}^{\prime}{ }^{\prime}-1 \\
\tilde{\phi}^{\prime}{ }_{N}
\end{array}\right]=\mathbf{R H S},
$$

where RHS is the vector of known terms defined on the right-hand-side of Eqs. (1-3). Equation (2) can be solved efficiently using the classic Thomas algorithm in a serial environment, but well-known difficulties arise in parallel.

In our approach to the domain-decomposition-based parallel environment, we assume that a 1-D domain discretised with $N$ nodes is decomposed into $M$ subdomains. Figure 1 shows the distribution of the nodes around the interface between subdomains $k$ and $k+1$, with $\phi_{i}, i=-4,-3, \cdots, N_{k}+4$ defined on subdomain $k$. Four halo nodes at each side of the subdomain are used to exchange data from neighboring subdomains, targeting a $6^{\text {th }}$-order 
central scheme. More halo nodes could also be defined for higher-order schemes.

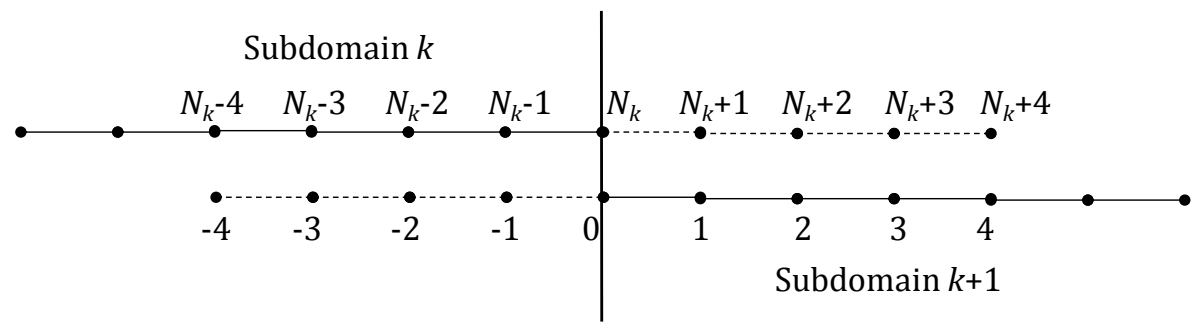

Figure 1: Schematic representation of the overlap region between two subdomains.

To decouple the compact scheme between subdomains $k$ and $k+1$, an explicit central scheme (ECS) with the same order of accuracy as the compact scheme is implemented for $i=-1$ and $N_{k}+1$ halo nodes to close the tridiagonal matrix. The procedure is shown in following Figure 2,

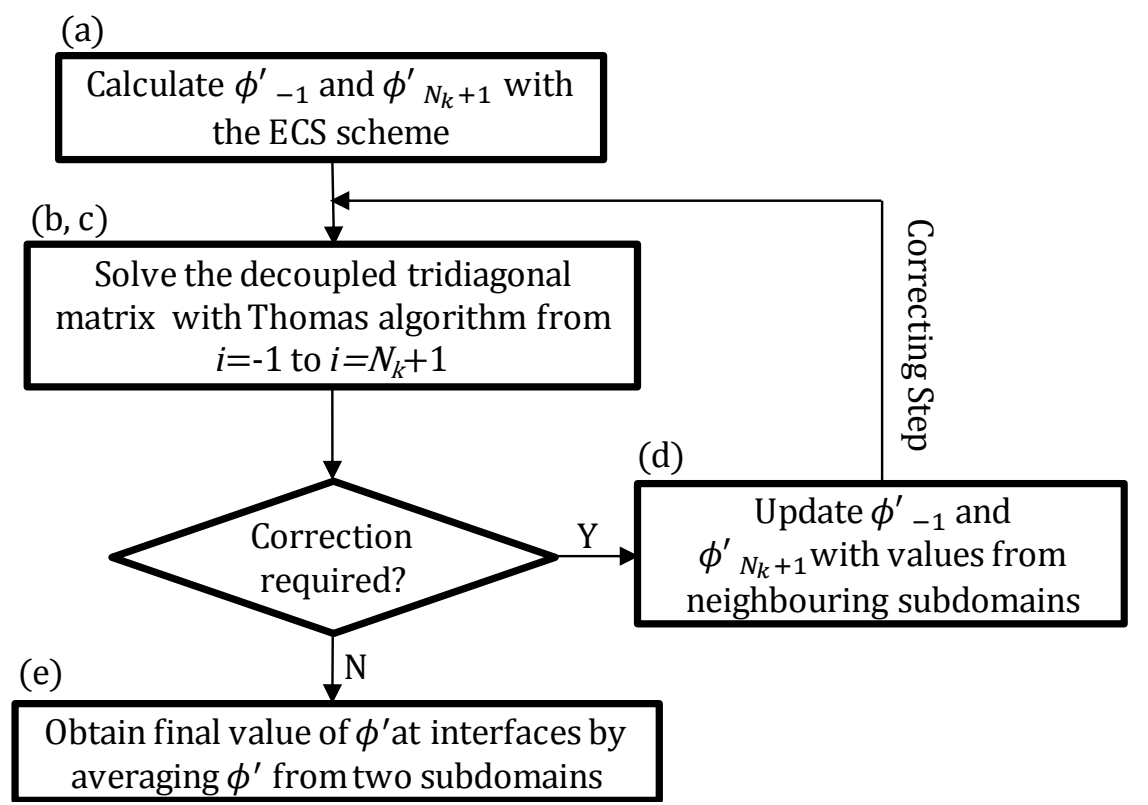

Figure 2: Procedure for the implementation of the DCOM scheme.

Its implementation is detailed as follows,

a) At the first layer of halo nodes, $i=-1$ and $N_{k}+1$, the ECS of the same order as the compact scheme is used to compute $\tilde{\phi}_{-1}^{\prime}$ and $\tilde{\phi}_{N_{k}+1}^{\prime}$ as,

$$
\tilde{\phi}_{-1}^{\prime}=\frac{1}{\Delta} \sum_{m=1}^{S^{*}} d_{m}\left(\phi_{-1+m}-\phi_{-1-m}\right)
$$

and

$$
\tilde{\phi}_{N_{k}+1}^{\prime}=\frac{1}{\Delta} \sum_{m=1}^{S^{*}} d_{m}\left(\phi_{N_{k}+1+m}-\phi_{N_{k}+1-m}\right),
$$

respectively, where $c_{m}$ is the coefficient defining an explicit central scheme, and $S^{*}$ is the half width of the stencil of the ECS scheme.

b) At the interface nodes, $i=0$ and $N_{k}$, Eq. (1) is reduced to, 


$$
\tilde{\phi}_{0}^{\prime}+\alpha \tilde{\phi}_{1}^{\prime}=\frac{1}{\Delta} \sum_{m=1}^{S} a_{m}\left(\phi_{m}-\phi_{-m}\right)-\alpha \tilde{\phi}_{-1}^{\prime}
$$

and

$$
\tilde{\phi}_{N_{k}}^{\prime}+\alpha \tilde{\phi}_{N_{k}-1}^{\prime}=\frac{1}{\Delta} \sum_{m=1}^{S} a_{m}\left(\phi_{N_{k}+m}-\phi_{N_{k}-m}\right)-\alpha \tilde{\phi}_{N_{k}+1}^{\prime}
$$

c) The tridiagonal matrix in subdomain $k$ is, therefore, closed as,

$$
\mathbf{A}_{\boldsymbol{k}}=\left[\begin{array}{ccccccc}
1 & \alpha & & & & \\
\alpha & 1 & \alpha & & & \\
& \alpha & 1 & \alpha & & \\
& & & \ddots & & & \\
& & \alpha & 1 & \alpha & \\
& & & & \alpha & 1 & \alpha \\
& & & & & \alpha & 1
\end{array}\right] .
$$

It can be solved independently with the classic Thomas algorithm within the subdomain.

d) Correction Step:

d1. Update $\tilde{\phi}_{-1}^{\prime}$ and $\tilde{\phi}_{N_{k}+1}^{\prime}$ by swapping data with neighboring nodes, e.g. exchanging $\tilde{\phi}_{N_{k}+1}^{\prime}$ in subdomain $k$ with $\tilde{\phi}_{1}^{\prime}$ in subdomain $k+1$.

d2. Repeat steps b) and c) to re-calculate the tridiagonal matrix and update $\tilde{\phi}_{i}^{\prime}$.

e) The final interface value is obtained by averaging $\tilde{\phi}_{N_{k}}^{\prime}$ from subdomain $k$ and $\tilde{\phi}_{0}^{\prime}$ from subdomain $k+1$.

In the above procedure, the correction steps (d) can be avoided or run many times, depending on the accuracy required and computing time available.

Unlike the scheme proposed by Sengupta et al. [26], no asymmetric schemes or operations near interdomain interfaces are introduced. Therefore, the spurious wave packets due to the asymmetry of the scheme can be avoided by the DCOM scheme.

Chao et al. [27] also adopted an ECS approach to decouple the corresponding compact scheme. They implement ECS on the interface nodes, i.e. $i=0$ and $i=N_{k}$ in Figure 1. Therefore, the benefit of the compact scheme is lost for interface nodes. The DCOM scheme implements the ECS approach on the halo nodes, i.e. $i=-1$ and $i=N_{k}+1$ and the scheme remains compact for all physical nodes. A comparison of the two schemes will be given in Section 3.

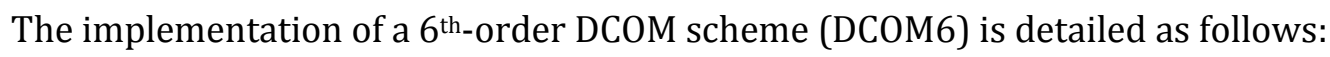

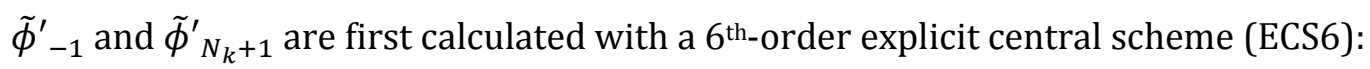

$$
\tilde{\phi}_{-1}^{\prime}=\frac{\phi_{2}-\phi_{-4}}{60 \Delta}-3 \frac{\phi_{1}-\phi_{-3}}{20 \Delta}+3 \frac{\phi_{0}-\phi_{-2}}{4 \Delta}
$$

and

$$
\tilde{\phi}_{N_{k}+1}^{\prime}=\frac{\phi_{N_{k}+4}-\phi_{N_{k}-2}}{60 \Delta}-3 \frac{\phi_{N_{k}+3}-\phi_{N_{k}-1}}{20 \Delta}+3 \frac{\phi_{N_{k}+2}-\phi_{N_{k}}}{4 \Delta} .
$$

At $i=0$ and $i=N_{k}$, the scheme reduces to, 


$$
\tilde{\phi}_{0}^{\prime}+\frac{1}{3} \tilde{\phi}_{1}^{\prime}=\frac{1}{36 \Delta}\left(\phi_{2}-\phi_{-2}\right)+\frac{7}{9 \Delta}\left(\phi_{1}-\phi_{-1}\right)-\frac{1}{3} \tilde{\phi}_{-1}^{\prime}
$$

and

$$
\frac{1}{3} \tilde{\phi}_{N_{k}-1}^{\prime}+\tilde{\phi}_{N_{k}}^{\prime}=\frac{1}{36 \Delta}\left(\phi_{N_{k}+2}-\phi_{N_{k}-2}\right)+\frac{7}{9 \Delta}\left(\phi_{N_{k}+1}-\phi_{N_{k}-1}\right)-\frac{1}{3} \tilde{\phi}_{N_{k}+1}^{\prime} .
$$

For internal nodes $\left(i=1,2, \cdots N_{k-1}\right)$ in the subdomain, the classic $6^{\text {th }}$-order compact central scheme (COM6) [7] is implemented,

$$
\frac{1}{3} \tilde{\phi}_{i-1}^{\prime}+\tilde{\phi}_{i}^{\prime}+\frac{1}{3} \tilde{\phi}_{i+1}^{\prime}=\frac{1}{36 \Delta}\left(\phi_{i+2}-\phi_{i-2}\right)+\frac{7}{9 \Delta}\left(\phi_{i+1}-\phi_{i-1}\right)
$$

Eqs. (12-14) is then solved independently in each subdomain using the Thomas algorithm against a closed tridiagonal matrix Eq. (9). At the correction step, $\tilde{\phi}_{-1}^{\prime}$ and $\tilde{\phi}_{N_{k}+1}^{\prime}$ are updated with values from the neighboring subdomain, and Eqs. (12-14) are recalculated using the updated $\tilde{\phi}_{-1}^{\prime}$ and $\tilde{\phi}_{N_{k}+1}^{\prime}$. The final interface value is acquired by averaging $\tilde{\phi}_{N_{k}}^{\prime}$ from subdomain $k$ and $\tilde{\phi}_{0}^{\prime}$ from subdomain $k+1$.

\subsection{Second derivative}

For the approximation of the $2^{\text {nd }}$ derivative, $\tilde{\phi}^{\prime \prime}$, the same principle as the $1^{\text {st }}$ derivative is applied to decouple the compact scheme. For brevity, we only give the formulas of the $6^{\text {th }}$ order DCOM scheme. The $6^{\text {th }}$-order compact and explicit schemes are respectively given as,

$$
\frac{2}{11} \tilde{\phi}^{\prime \prime}{ }_{i-1}+\tilde{\phi}^{\prime \prime}{ }_{i}+\frac{2}{11} \tilde{\phi}^{\prime \prime}{ }_{i+1}=3 \frac{\phi_{i+2}-2 \phi_{i}+\phi_{i-2}}{44 \Delta^{2}}+12 \frac{\phi_{i+1}-2 \phi_{i}+\phi_{i-1}}{11 \Delta^{2}},
$$

and

$$
\tilde{\phi}^{\prime \prime}{ }_{i}=\frac{\phi_{i+3}-2 \phi_{i}+\phi_{i-3}}{90 \Delta^{2}}-3 \frac{\phi_{i+2}-2 \phi_{i}+\phi_{i-2}}{20 \Delta^{2}}+3 \frac{\phi_{i+1}-2 \phi_{i}+\phi_{i-1}}{2 \Delta^{2}} .
$$

The same idea is applied to decouple Eq. (15) at the interface $i=0$ as,

$$
\tilde{\phi}^{\prime \prime}{ }_{0}+\frac{2}{11} \tilde{\phi}_{1}^{\prime \prime}{ }_{1}=3 \frac{\phi_{i+2}-2 \phi_{i}+\phi_{i-2}}{44 \Delta^{2}}+12 \frac{\phi_{i+1}-2 \phi_{i}+\phi_{i-1}}{11 \Delta^{2}}-\frac{2}{11} \tilde{\phi}^{\prime \prime}{ }_{-1},
$$

and $\tilde{\phi}^{\prime \prime}{ }_{-1}$ is calculated with the explicit scheme given by Eq. (16). A symmetric operation is implemented for $i=N_{k}$ and $i=N_{k}+1$. At the last step, the interface values from two neighbouring subdomains are averaged as the final interface values.

\section{Analysis of numerical schemes}

\subsection{Fourier analysis}

The spectral properties of the proposed schemes can be investigated using Fourier analysis. For a pure harmonic function,

$$
\phi(x)=e^{i \omega x},
$$

where $i=\sqrt{-1}$ is the imaginary unit, and $\omega$ is the wavenumber. The $1^{\text {st }}$ and $2^{\text {nd }}$ derivatives 
of $\phi(x)$ are respectively expressed as,

$$
\phi^{\prime}(x)=i \omega e^{i \omega x}=i \omega \phi(x)
$$

and

$$
\phi^{\prime \prime}(x)=-\omega^{2} e^{i \omega x}=-\omega^{2} \phi(x) .
$$

The approximation of $\phi^{\prime}(x)$ and $\phi^{\prime \prime}(x)$ by a finite difference scheme can be expressed in the modified wavenumber as,

$$
\tilde{\phi}^{\prime}=i \widehat{\omega} \phi
$$

and

$$
\tilde{\phi}^{\prime \prime}=-\omega^{\prime \prime} \phi
$$

The relationship between $\widehat{\omega}$ and $\omega^{\prime \prime}$ with $\omega$ can therefore be reduced to,

$$
\widehat{\omega}=-i \tilde{\phi}^{\prime} / \phi
$$

and

$$
\omega^{\prime \prime}=-\tilde{\phi}^{\prime \prime} / \phi
$$

for the $1^{\text {st }}$ and $2^{\text {nd }}$ derivatives, respectively.

The implementation of the DCOM scheme is related to the number of nodes in the subdomain, data swapping and averaging between neighbouring subdomains, and analytical expressions for $\widehat{\omega}$ and $\omega^{\prime \prime}$ are therefore hard to obtain. To analyse its spectral property, the numerical approximations of the $1^{\text {st }}$ and $2^{\text {nd }}$ derivatives of $\phi(x)$ (i.e. $\tilde{\phi}^{\prime}$ and $\left.\tilde{\phi}^{\prime \prime}\right)$, with $\omega$ ranging from 0 to $\pi$, are calculated by specific schemes in a 1-D domain $x \in$ $[0,2 \pi]$ discretised with $N$ nodes. These nodes are distributed in $M$ subdomains, and $\widehat{\omega}$ and $\omega^{\prime \prime}$ are calculated by using Eqs. (23) and (24), respectively. The analysis of mean values for $\widehat{\omega}$ and $\omega^{\prime \prime}$, averaged on all nodes, and the values of $\widehat{\omega}$ and $\omega^{\prime \prime}$ on the interface node are conducted. It is necessary to clarify that the Fourier analysis here is only for inner nodes, and the influence of boundary constrains, which has been well discussed previously $[7,49,50]$, is not taken into account.

The real part of the modified wavenumber, $\widehat{\omega}$, for the $1^{\text {st }}$ derivatives with $N=64$ are shown in Figure 3 (a). It considers subdomain number $M$ equal to 4 and 16, corresponding to $N_{k}=16$ and $4\left(N_{k}=N / M\right)$. As expected, the compact schemes show higher resolution at large wavenumbers than the explicit scheme of the same order. Chao's scheme, shown in Figure 3, refers to the $6^{\text {th }}$-order decoupled compact scheme (see Chao et al. [27]). It falls between those of ECS6 and COM6, and approaches that of COM6 as $N_{k}$ is increased. The DCOM6 scheme proposed in the present paper shows a better spectral performance than Chao's scheme. Its spectral property is closer to that of the COM6 scheme and is less sensitive to the number of subdomains. Tests with $N_{k}=4$ show that the implementation of the 
correction step can be used to effectively improve the accuracy of the DCOM scheme. The imaginary parts of $\widehat{\omega}$ for all tested schemes are zero (not shown here), indicating their nondissipative nature due to the symmetric form of the schemes. This is important in DNS/LES of turbulence, because the numerical dissipation has a critical effect on the prediction of kinetic energy of turbulent eddies. [51,52,53,54]

The spectral properties of the schemes at the interface node are presented in Figure 3 (b). At the interface node, Chao's scheme degrades to the ECS approach, since the ECS scheme is used to acquire $\tilde{\phi}^{\prime}$ at the interface. For the DCOM6 scheme without a correction step, a clear improvement over Chao's scheme is observed, and the DCOM6 scheme with the correction step shows an almost identical result to the fully coupled COM6 scheme.

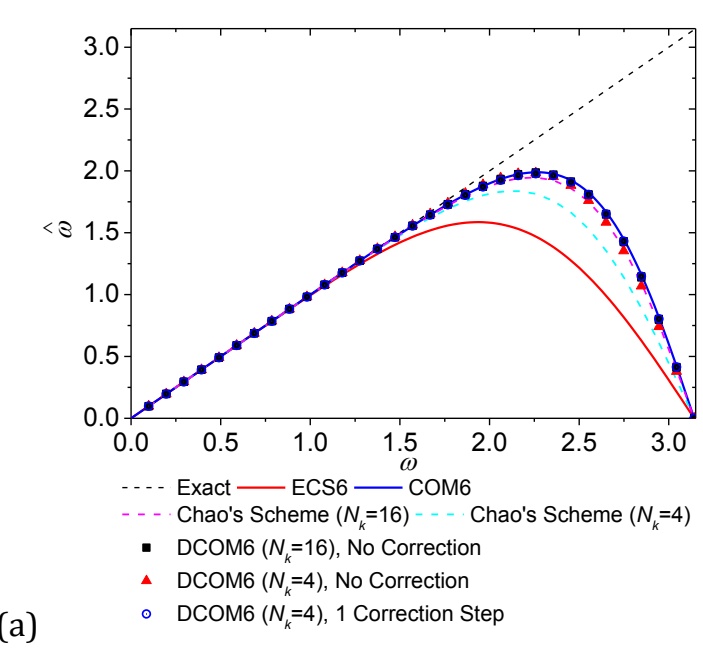

Figure 3: Mean (a) and Interface (b) values of $\widehat{\omega}$ (real part) of $6^{\text {th }}$-order central schemes for the $1^{\text {st }}$ derivative.

The error in the $2^{\text {nd }}$ desrivative approximation is measured as $\left|\omega^{\prime \prime}-\omega^{2}\right|$ [7] and presented in Figure 4. Similar to the observation of the $1^{\text {st }}$ derivatives, the mean value of $\left|\omega^{\prime \prime}-\omega^{2}\right|$ of the DCOM6 scheme is very close to that of the COM6 scheme, even for the case with $N_{k}=4$, and without any correction step. At the interface node, Chao's scheme collapses again onto the curve of the EXP6 scheme, and the curve of the DCOM6 scheme without the correction step is close to the COM6 scheme. The inclusion of the correction step further improves the result towards the COM6 scheme. 

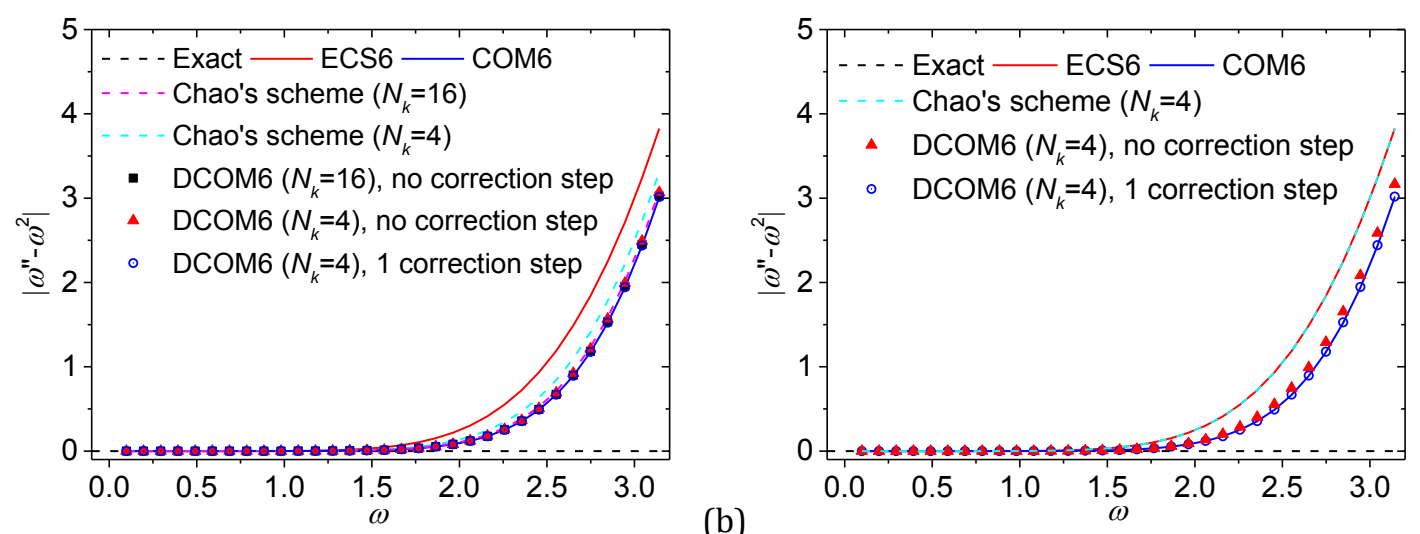

Figure 4: Mean (a) and Interface (b) values of $\left|\omega^{\prime \prime}-\omega^{2}\right|$ of $6^{\text {th }}$-order central schemes for the $2^{\text {nd }}$ derivative.

It can be seen that the proposed DCOM6 scheme possesses superior characteristics in wavenumber space compared to Chao's scheme, and its performance is close to the fullycoupled compact scheme.

\subsection{Error analysis}

The numerical error of a scheme can be evaluated by calculating the $1^{\text {st }}$ and $2^{\text {nd }}$ derivatives of the following test function:

$$
f(x)=\sin (4 x), x \in[0,2 \pi]
$$

and the $l_{2}$-errors against the exact solution. The $l_{2}$-errors is defined as $\sqrt{\frac{1}{N} \sum_{i=1}^{N}\left(\hat{f}_{i}^{\prime}-f^{\prime}\right)^{2}}$, where $\hat{f}_{i}^{\prime}$ is the approximation of $f^{\prime}$ at node $i$, and $N$ is the number of nodes.

The $l_{2}$-errors of the approximation of the $1^{\text {st }}$ derivative against both the number of nodes and the number of subdomains are presented in Figure 5. It can be seen in Figure 5(a) that

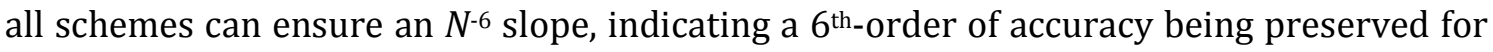
all schemes. The $l_{2}$-error of the DCOM6 scheme with one correction step overlaps that of the COM6 scheme. The DCOM6 scheme without any correction step contains larger errors than with the correction step but smaller errors than Chao's scheme. According to Figure 5(b), the level of errors for all tested decoupled schemes has shown a trend of approaching the COM6 scheme as the number of nodes in each subdomain increases (i.e. the degree of parallelism decreases). Once again, the DCOM6 scheme with the correction step has shown similar performance to the COM6 scheme, and the DCOM6 scheme without a correction step gives smaller errors than Chao's scheme. 


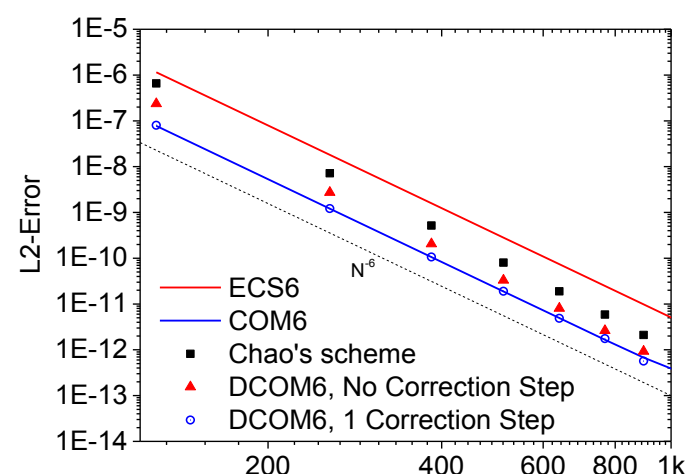

(a)

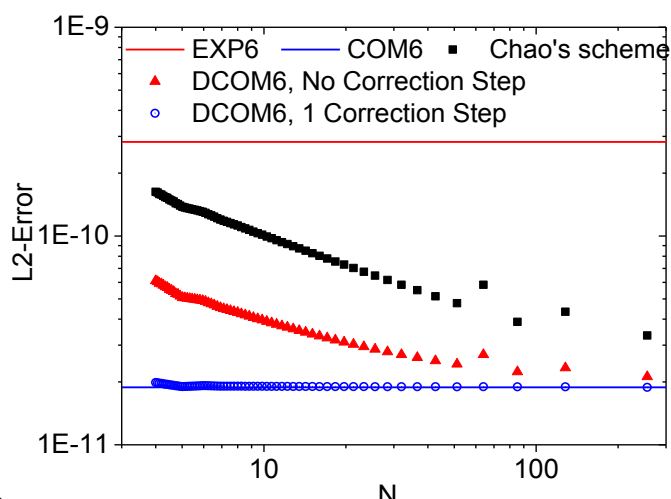

(b)
$\mathrm{N}_{\mathrm{k}}$

Figure 5: $l_{2}$-errors of the approximation of the $1^{\text {st }}$ derivative using $6^{\text {th }}$-order schemes. (a) is based on the number of subdomains $\mathrm{M}=32$ and (b) is based on the total number of nodes $\mathrm{N}=512$.

Figure 6 plots the $l_{2}$-errors of the approximation of the $2^{\text {nd }}$ derivative using $6^{\text {th }}$-order schemes. All schemes tested achieve $6^{\text {th }}$-order accuracy, although errors increase after their values are reduced to $10^{-10}$, due to machine round-off error. Note that the DCOM6 schemes with and without the correction step produce almost the same error as that of the COM6 scheme. In Figure 6 (b), with the increase of $N_{k}, l_{2}$-errors of both DCOM6 and Chao's schemes fall to the value of the COM6 scheme, although Chao's scheme generates larger errors than the DCOM6 scheme at small $N_{k}$.

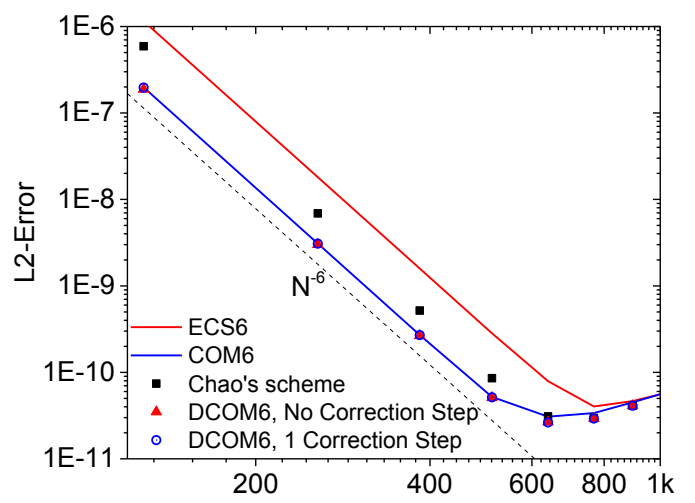

(a)

Figure 6: $l_{2}$-error of the approximation of the $2^{\text {nd }}$ derivative using $6^{\text {th }}$-order schemes. (a) is based on the number of subdomains $M=32$ and (b) is based on the total number of nodes $N=512$.

\section{Numerical tests}

In this section, the DCOM6 schemes are tested in canonical cases.

\subsection{1-D wave-packet propagation}

The performance of the DCOM6 schemes is now tested for a 1-D wave-packet propagation problem, and compared with the ECS6, COM6 and Chao's schemes. The 1-D linear convection equation is solved as,

$$
\frac{\partial u}{\partial t}+c \frac{\partial u}{\partial x}=0
$$


where $u$ is a time-spatial variable, and $t$ and $x$ are the time and spatial coordinates, respectively.

The equation describes the propagation of the initial wave-packet with a constant speed, $c$, and it has been widely used to study the dispersion property of numerical schemes [26, 55]. By referencing the numerical test done by Sengupta et al. [26], the convection speed is set as $c=0.5$, and the initial wave-packet is given as,

$$
u(x, 0)=e^{-A\left(x-x_{0}\right)^{2}} \sin \left(\omega_{0} x\right),
$$

in which $A=50, x_{0}=1.5$, and $\omega_{0}=0.838242 / \Delta x, \Delta x$ is the size of the mesh. The computational domain is $x \in[0,10]$ with periodic conditions applied at both ends. A 1-D uniform mesh with 664 nodes is used to solve Eq. (26). A four-step Runge-Kutta scheme [56] is implemented as the time integration strategy because of its good numerical stability and dispersion relation preservation property. The time step is set as $\Delta t=0.0006$.

The profiles of $u$ at $t=0,3,6,9,12$, and 15 by the ESC 6 scheme are shown in Figure 7 (a). The spurious wiggles (i.e. the so-called q-waves) on the left-hand side of the wave-packet are produced by the dispersive error of the numerical method [57], and they grow and spread upstream with time. By comparing the results of different schemes at $t=15$ in Figure 7 (b), we can see that the spurious wiggles are effectively removed by using compact schemes, including both Chao's scheme and the DCOM6 scheme, due to their higher resolution and smaller dispersive errors at high wavenumbers, as presented in Figure 3. The results of compact schemes lie on top of the exact solution in Figure 7 (b). A detailed analysis will be presented later.

(a)

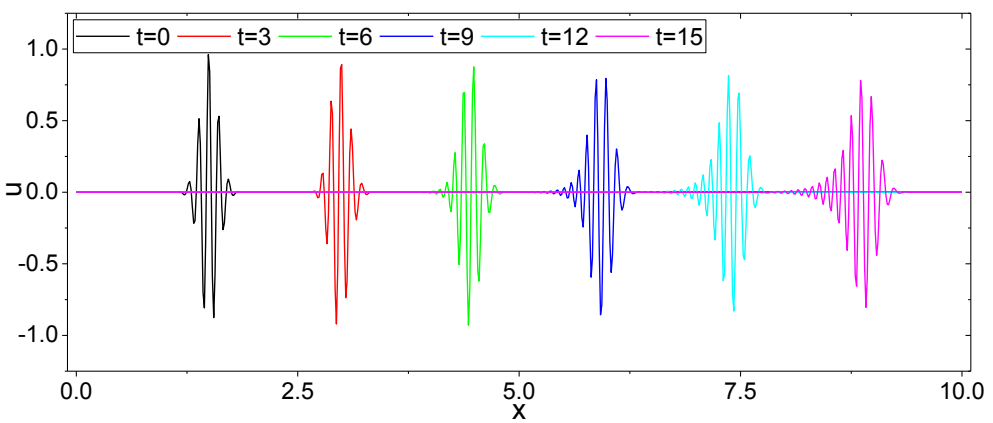

(b)

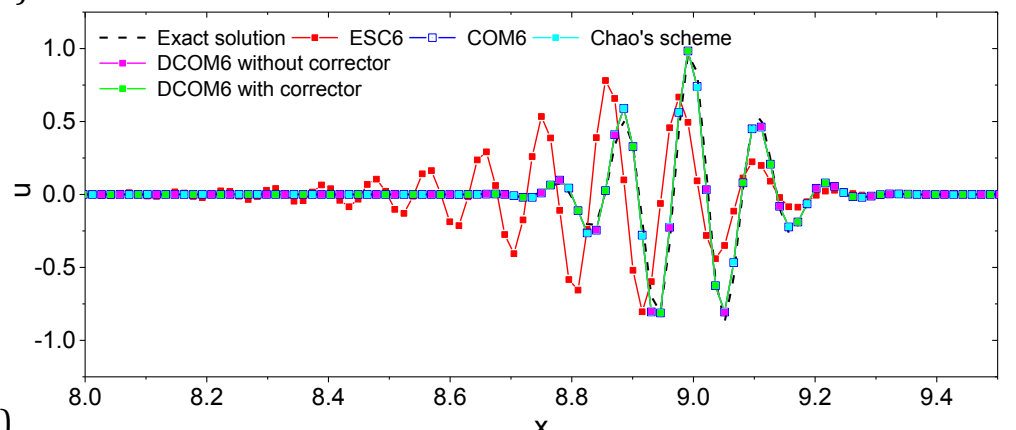

Figure 7: Capture of wave-packet from $t=0$ to $t=15$ by ECS6 scheme, and the comparison of results from different scheme. For the tests of parallel schemes, the computational domain is split into 4 even subdomains, and each subdomain has 166 nodes. 
The temporal evolution of the results from Chao's scheme with the largest degree of parallelism permitted is presented in Figure 8 (a), in which the computational domain is split into 166 subdomains and each subdomain contains only 4 nodes. It can be observed that with a large degree of the parallelism, the results from Chao's scheme have deteriorated, and strong wiggles are generated on the left-hand side of the wave-packet, similar to the result of the ECS6 scheme shown in Figure 7(a). Moreover, weak wiggles appear across the whole domain for the result at $t=15$. By comparing the results of Chao's and the DCOM schemes at $t=15$ in Figure 8 (a), it can be confirmed that the DCOM6 scheme shows much improved results, and the scheme, even without the correction step, shows only very weak wiggles at $\mathrm{t}=15$, and the correction step removes the wiggles very effectively.

(a)
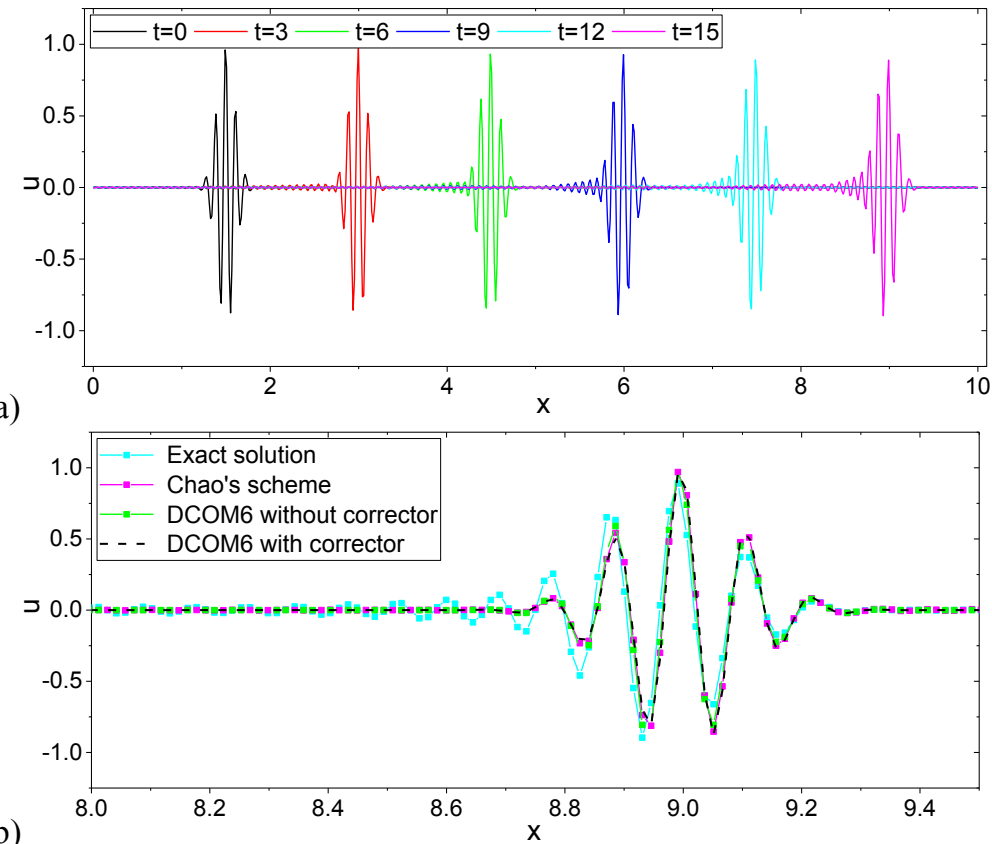

Figure 8: The propagation of the wave-packet by Chao's scheme (a), and comparison to the results from the decoupled schemes (b). The computational domain is split into 166 even subdomains.

By examining the details of the left-hand side of the wave-packet at $t=15$ in Figure 9, weak wiggles generated by Chao's scheme are observed for the case with 4 subdomains, and the wiggles are amplified with an increase in the number of subdomains. The DCOM6 scheme shows a more stable performance, and the correction step can further recover the result to that of the COM6 scheme.

The $I_{2}$-errors, $\sqrt{\frac{1}{N} \sum_{i=1}^{N}\left(u_{i}-u_{\text {exact }}\right)^{2}}$, for the solution at $t=20$ against the number of nodes per subdomains are presented in Figure 10, in which $u_{\text {exact }}(x, 20)=u(x, 0)$. For this test, the total number of nodes is set to 512 so that the nodes can be evenly distributed. It can be confirmed that the DCOM6 scheme has smaller errors than Chao's scheme and the correction step can restore the DCOM6 scheme to the accuracy of the fully-coupled compact scheme. It is interesting to note that the DCOM6 scheme, without any correction step, can 
produce even smaller errors than the COM6 scheme, and the errors decrease with the decrease of the number of nodes per subdomain. By examining the distribution of errors, defined as $\left|u_{i}-u_{\text {exact }}\right|$ at $t=20$ for the case with 8 nodes per subdomain, in Figure 11, we can see the DCOM6 scheme without a correction step produces smaller errors within the wave-packet centred at $\mathrm{x}=1.5$ which makes the $l_{2}$-errors being smallest among all tested schemes, although wiggles can be observed on the profile away from the wave-packet. Since the value of $u$ is close to zero away from the wave-packet, the ratio of the errors to the local value of $u$ is much bigger than the COM6 scheme. From Figure 11 we can see similar results between the DCOM6 scheme with the correction step and the fully-coupled COM scheme, since the profiles from these two schemes lie on top of each other.
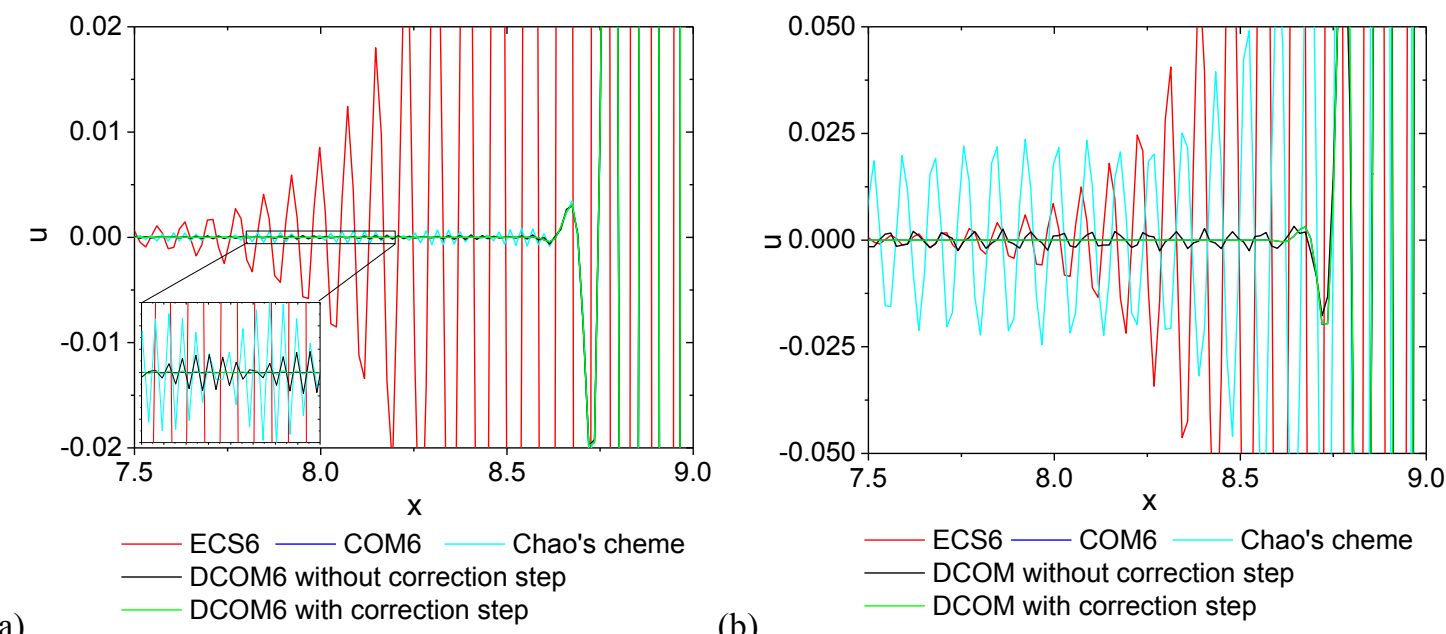

Figure 9: Detailed comparison of the wave-packets at $t=15$. The computational domain is split into 4 and 166 subdomains in (a) and (b) respectively.

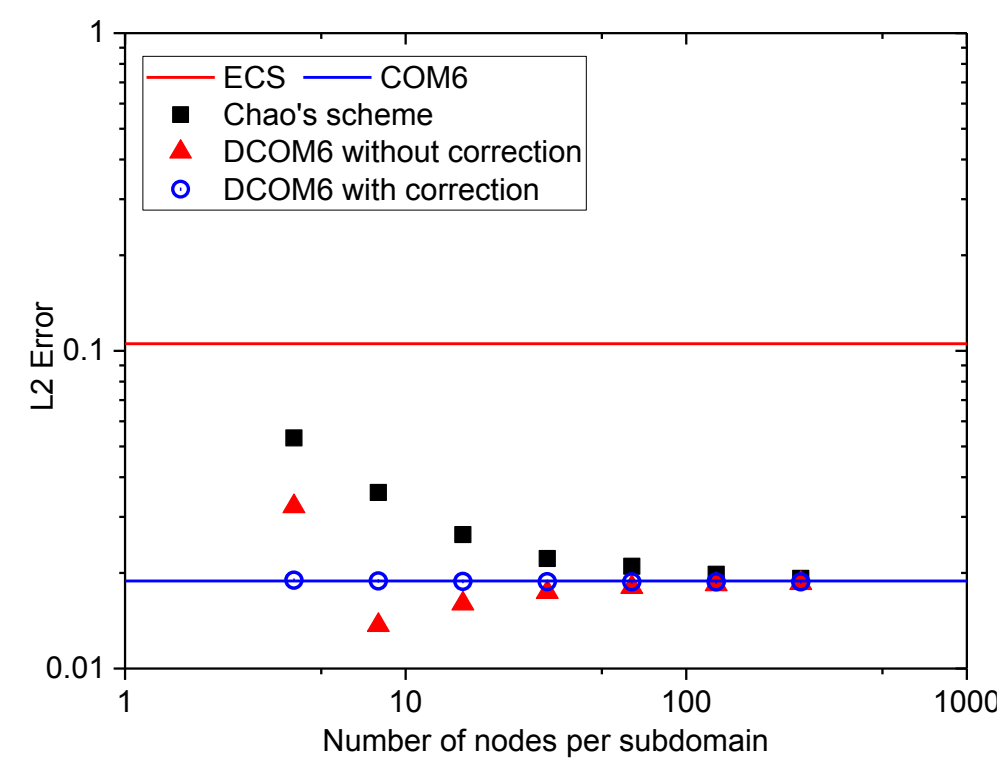

Figure 10: $l_{2}$-norm errors of results of different schemes at $\mathrm{t}=20$. 


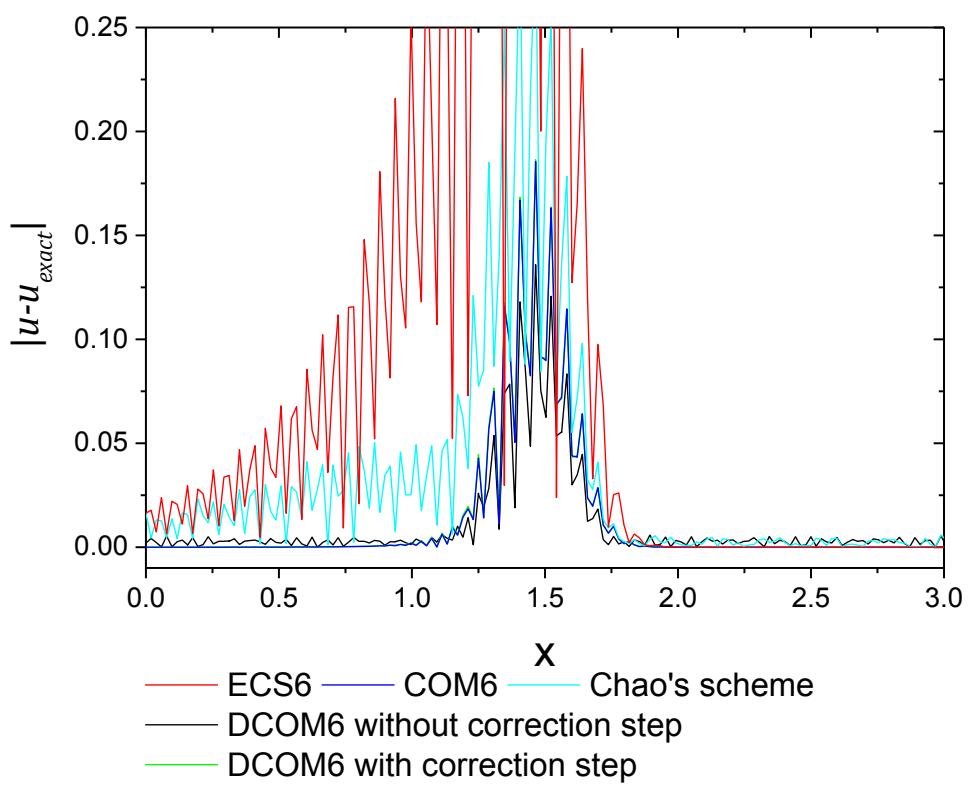

Figure 11: Distributions of errors of results at $t=20$ for the case with 64 subdomains.

\subsection{2-D vorticity wave convection problem}

The DCOM6 scheme is further tested on a 2-D vorticity wave convection in a subsonic freestream by solving the 2-D Euler equations,

$$
\begin{gathered}
\frac{\partial \rho}{\partial t}+\frac{\partial \rho u_{i}}{\partial x_{i}}=0 \\
\frac{\partial \rho u_{i}}{\partial t}+\frac{\partial\left(\rho u_{i} u_{j}+p \delta_{i j}\right)}{\partial x_{j}}=0 \\
\frac{\partial \rho e}{\partial t}+\frac{\partial(\rho e+p) u_{j}}{\partial x_{j}}=0
\end{gathered}
$$

in which the primary variables are the density $\rho$, the pressure $p$, the velocity component $u_{i}$, and the total energy per unit mass $e$, defined as, $e=\frac{p}{(\gamma-1) \rho}+\frac{1}{2} u_{i} u_{i}$, with the ratio of specific heats $\gamma=1.4$. The standard Einstein summation notation is used and notations $x_{i}$ and $u_{i}$, $i=1,2$ are adopted to represent $(x, y)$ and $(u, v)$, respectively. The Euler equations are non-dimensionalised with the freestream density, $\rho_{0}$, velocity, $u_{0}$, temperature, $T_{0}$, and the reference length, $L$.

The convective terms are solved in the skew-symmetric form to reduce the aliased errors arising from nonlinear terms $[58,59,60,61,62]$. The time integration is conducted using the classic 3-step 3rd-order TVD Runge-Kutta scheme [63]. A 2-D domain with size $L_{x} \times L_{y}=[0,20] \times[0,10]$ is discretised with a $64 \times 32$ uniform mesh, and periodic boundary conditions are applied at all boundaries. The initial conditions are, 
Accepted by International Journal for Numerical Methods in Fluids

$$
\begin{gathered}
\rho=1 \\
u=1-C_{v s} \frac{y-y_{c}}{R_{v s}^{2}} e^{-r^{2} / 2}, \\
v=C_{v s} \frac{x-x_{c}}{R_{v s}^{2}} e^{-r^{2} / 2}, \\
p=p_{0}-0.5 \frac{C_{v s}^{2}}{R_{v s}^{2}} e^{-r^{2}},
\end{gathered}
$$

with $x_{c}=10$ and $y_{c}=5$ as the initial location of the vortical core, the freestream pressure is given as, $p_{0}=1 / \gamma M a^{2}$, and the size and strength of the initial vortex is specified with $R_{v s}=1$ and $C_{v s}=0.01 u_{0} R_{v s}$. The freestream Mach number is, $M a=u_{0} / \sqrt{\gamma R T_{0}}=0.1$, with the gas constant $R=287.1 \mathrm{~J} /(\mathrm{K} \cdot \mathrm{kg})$. This case has been widely adopted to test numerical schemes with respect to vortex preservation $[8,38,64,65,66]$. The exact solution of the equations at time $t$ can be obtained by shifting the initial solution by $u_{0} t$ in the $x$-direction [38].

The computational domain was split into $16 \times 8$ subdomains for parallel computations, as shown in Figure 12 , and each subdomain contains $4 \times 4$ nodes. The vorticity field, given by $\omega=\partial v / \partial x-\partial u / \partial y$, at $\mathrm{t}=20$, obtained from the DCOM scheme with the corrector is shown in Figure 12, from which we can see that the vortex is well resolved. The temporal evolution of $l_{2}$-errors are presented in Figure 13 where the $l_{2}$-error here is defined as $\frac{1}{\omega_{\max }} \sqrt{\frac{1}{N_{i} N_{j}} \sum_{j=1}^{N_{j}} \sum_{i=1}^{N_{i}}\left(\omega_{i, j}-\omega_{\text {exact }}\right)^{2}}$, in which $\omega_{i, j}$ is the vorticity on the node $(i, j), \omega_{\text {exact }}$ is the exact solution of the vorticity, $\omega_{\max }$ is the maximum of the initial vorticity, and $N_{i}$ and $N_{j}$ are the nodes' numbers in the $x$ and $y$-directions, respectively.

The proposed DCOM6 scheme produces smaller errors than Chao's scheme, as expected. For Chao's scheme, errors increase with an increase in the degree of parallelism, and the DCOM6 scheme gives a relatively stable performance, especially with the correction step. Once again, the decrease of numerical errors with an increase in subdomain number for the DCOM6 scheme, without any correction step, is observed. By examining the distribution of errors, $\left|\omega_{i, j}-\omega_{\text {exact }}\right|$, in Figure 14, we can confirm that the errors of the ECS6 scheme is the highest, and Chao's scheme induces errors across the entire domain. The DCOM6 scheme without a correction step gets the smallest errors around the vortex but it generates ripples outside of the vortex. The implementation of the correction step causes higher errors within the vortex, but it gives a clean result in the rest of the domain. Therefore, the quality of the result of the DCOM6 scheme with the correction step is regarded as the best. 
Accepted by International Journal for Numerical Methods in Fluids

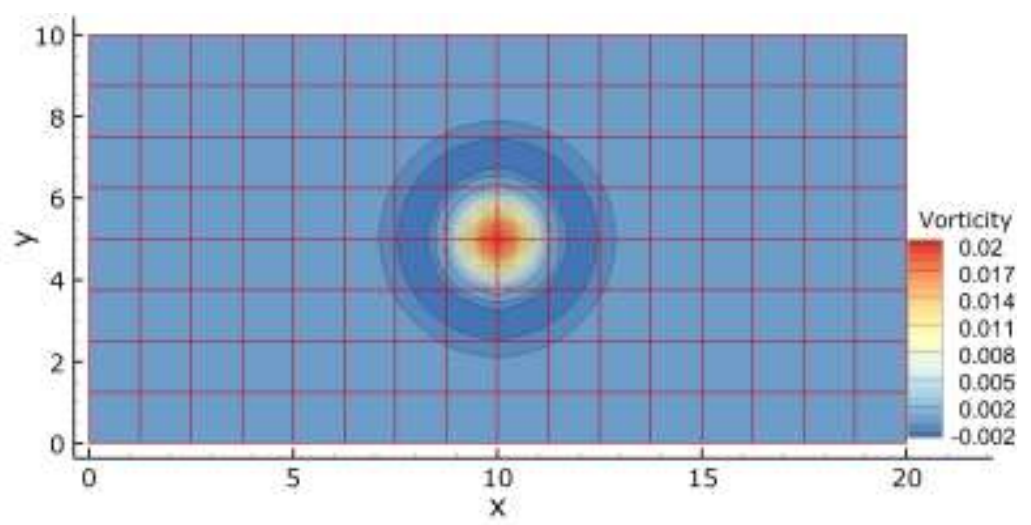

Figure 12: Vorticity field at $\mathrm{t}=20.0$ by the DCOM6 with correction step. The mesh lines mark the interdomain interfaces.
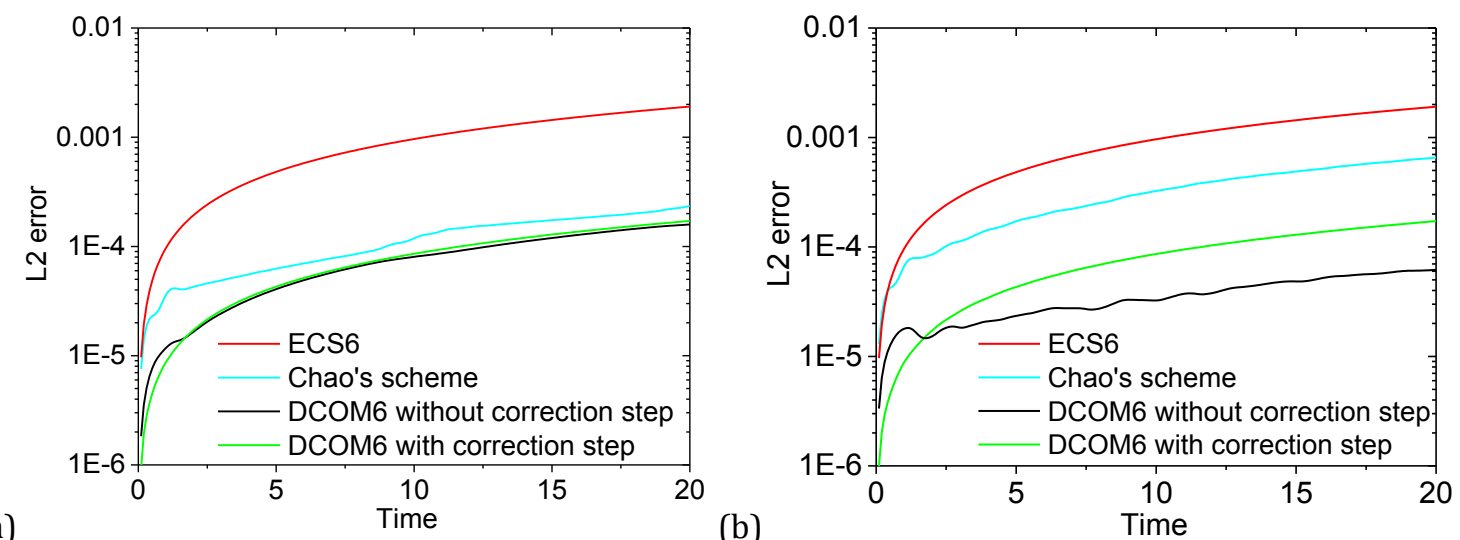

Figure 13: Temporal evolution of $l_{2}$-norm errors. (a): 4 subdomains; (b): 128 subdomains.

(a)

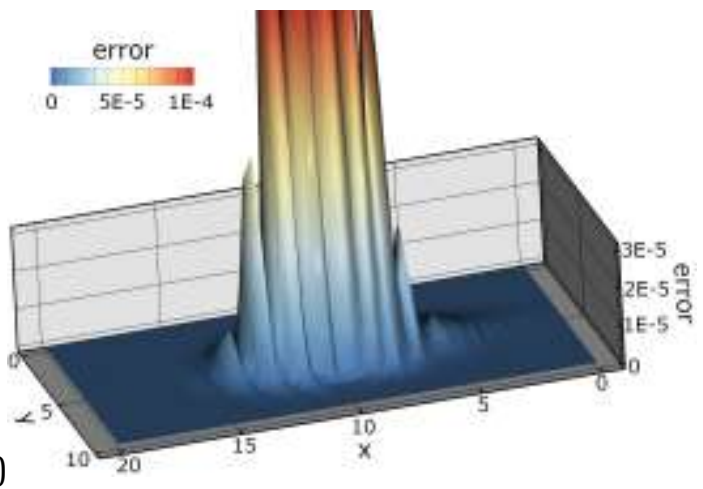

(b)

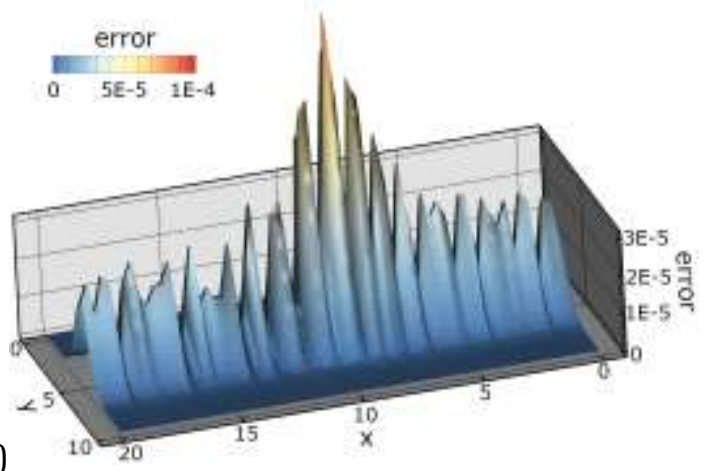

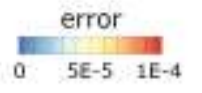

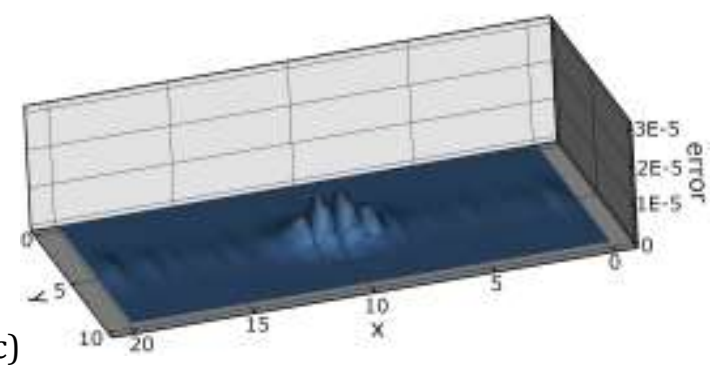

(d)

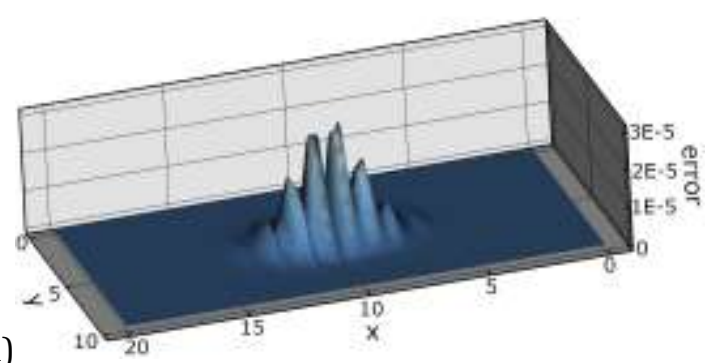

Figure 14: Distribution of errors at $\mathrm{t}=20$ with 128 subdomains. (a): ECS6 scheme; (b): Chao's scheme; (c): DCOM6 scheme without correction step; (d): DCOM scheme with the correction step. 


\section{Application of the DCOM6 scheme to turbulence simulations}

In this section, the DCOM6 scheme is applied to solve the 3-D Navier-Stokes (N-S) equations with an in-house finite-difference solver, ASTR, for two classic problems: the 3-D Taylor-Green vortex problem and planar turbulent channel flow to show its capability in DNS of turbulent flows. The 3-D N-S equations are given in non-dimensional form as,

$$
\begin{gathered}
\frac{\partial \rho}{\partial t}+\frac{\partial \rho u_{i}}{\partial x_{i}}=0 \\
\frac{\partial \rho u_{i}}{\partial t}+\frac{\partial\left(\rho u_{i} u_{j}+p \delta_{i j}\right)}{\partial x_{j}}=\frac{\partial \sigma_{i j}}{\partial x_{j}}+s_{i} \\
\frac{\partial \rho e}{\partial t}+\frac{\partial(\rho e+p) u_{j}}{\partial x_{j}}=\frac{\partial\left(\sigma_{i j} u_{i}-q_{j}\right)}{\partial x_{j}}+s_{e}
\end{gathered}
$$

The stress tensor and the heat flux vector are expressed as,

$$
\sigma_{i j}=\frac{\mu}{\operatorname{Re}}\left(\frac{\partial u_{i}}{\partial x_{j}}+\frac{\partial u_{j}}{\partial u_{i}}-\frac{2}{3} \delta_{i j} \frac{\partial u_{k}}{\partial x_{k}}\right)
$$

and

$$
q_{i}=-\frac{\mu}{\operatorname{PrRe}(\gamma-1) M a^{2}} \frac{\partial T}{\partial x_{i}}
$$

The non-dimensional parameters which appear on the stress and the heat flux are the Reynolds number and Prandtl number, $\operatorname{Re}=\rho_{0} u_{0} L / \mu_{0}$ and $\operatorname{Pr}=\mu C_{P} / \vartheta=0.72$, where $C_{P}=\gamma R /(\gamma-1)$ is the specific heat capacity of the gas at constant pressure, and $\vartheta$ is thermal conductivity coefficient. The dynamic viscosity coefficient, $\mu$, is calculated via Sutherland's law,

$$
\mu=T^{1.5} \frac{T_{S} / T_{0}+1}{T+T_{S} / T_{0}} .
$$

According to the analysis in Section 2, the DCOM scheme with one correction step gives the best result, and the DCOM scheme without any correction is considered as the second best. However, the computing time using the DCOM scheme with one correction step is about twice the DCOM without any correction step. Therefore, for the trade-off between accuracy and computing time, the correction step is not implemented for simulations of 3D turbulent flows in the present paper.

The convective terms and time integration are solved in the same way as the Euler equations Eq. (28). The diffusive terms are solved in the Laplacian form [67] to improve the stability of the solver. The $2^{\text {nd }}$ derivatives in the diffusion terms, i.e. $\partial^{2} u_{i} / \partial x_{j}^{2}$ and $\partial^{2} T / \partial x_{j}^{2}$, are also calculated by using the DCOM6 scheme for $2^{\text {nd }}$ derivatives Eq. (15-17).

\subsection{3-D Taylor-Green vortex problem}


The DCOM6 scheme is implemented for the 3-D Taylor-Green Vortex (TGV) problem [68] by solving the $\mathrm{N}-\mathrm{S}$ equations in a $2 \pi$ cubic domain. A $256^{3}$ uniform mesh is used to discretise the domain and the computational domain is split into $768(12 \times 8 \times 8)$ subdomains.

The initial condition is set as $[69,70]$,

$$
\begin{gathered}
\rho=1, \\
u=\sin (x) \cos (y) \cos (z), \\
v=-\cos (x) \sin (y) \cos (z), \\
w=0, \\
p=1 / \gamma M^{2}+\frac{1}{16}[\cos (2 x)+\cos (2 y)][\cos (2 z)+2] .
\end{gathered}
$$

The reference Mach number is $M a=0.1$ and the reference Reynolds number is 1600 .

The evolution of the spatially averaged kinetic energy and enstrophy obtained with the DCOM6 schemes is compared to that obtained with a pseudo-spectral method [71] on a $512^{3}$ mesh, shown in Figure 15. Both the kinetic energy and enstrophy profiles agree well with the data from the pseudo-spectral code, although a slight underprediction of the enstrophy peak value by the DCOM6 schemes can be observed. The 3-D fields of enstrophy and coherent structures visualised with iso-surfaces of $\lambda_{c i}$ at $\mathrm{t}=0,10$, and 20 are shown in Figure 16 and Figure 17, respectively. The imaginary part of the complex eigenvalue of the velocity gradient tensor $\lambda_{c i}$ was first proposed by Zhou et al. [72] to visualise turbulent coherent structures, and it was then known as swirling strength [10], and widely adopted as a vortex identification scheme $[73,74]$. It can be seen that the flow structures evolve into smaller vortical structures with higher vorticity from $t=0$ to $t=10$, and from $t=10$ to $t=20$, the vortical structures become more chaotic, occupying a greater space, although the maximum strength of the enstrophy is reduced, indicating a dissipation and diffusion process. At $t=0,10$, and 20 , the symmetry of the flow field is well preserved.
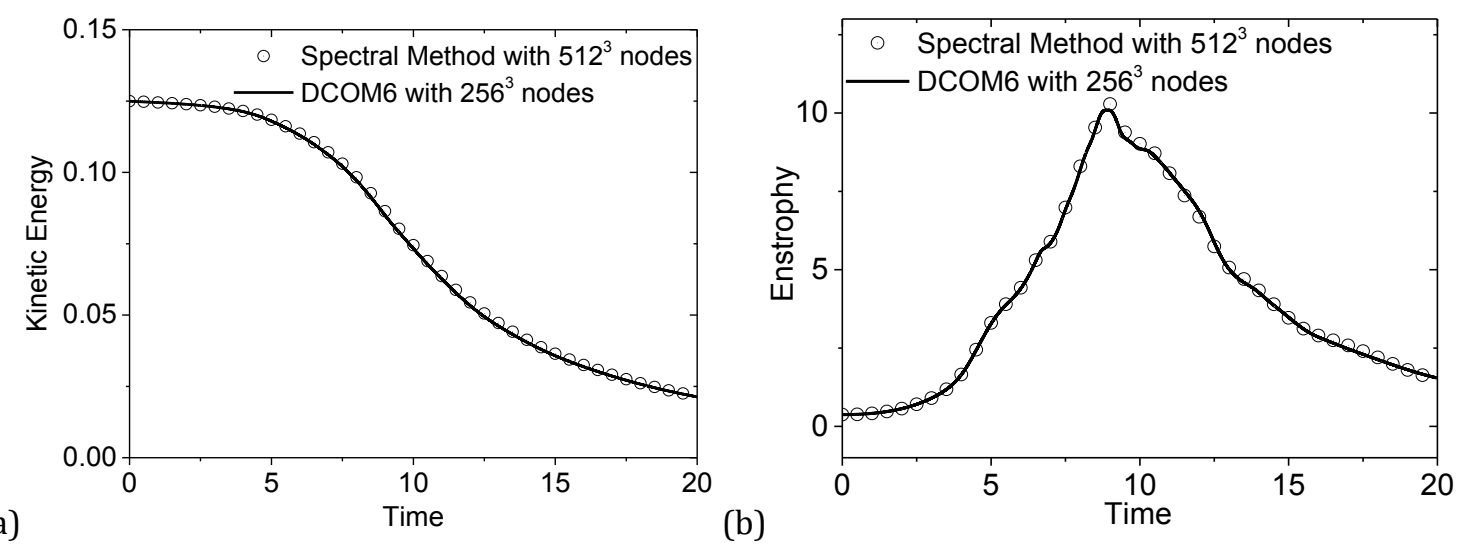

Figure 15: Temporal evolution of kinetic energy (a) and enstrophy (b) 
(a)

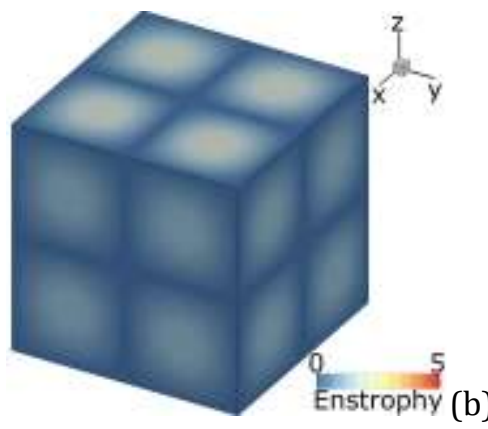

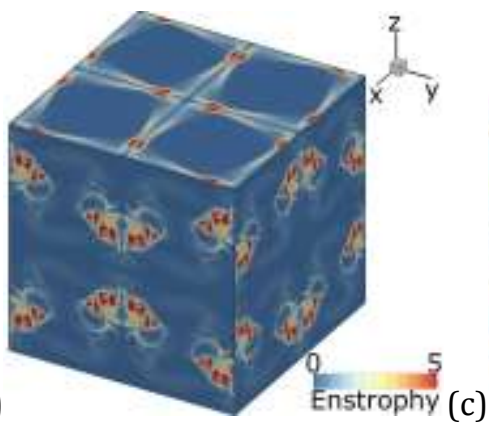

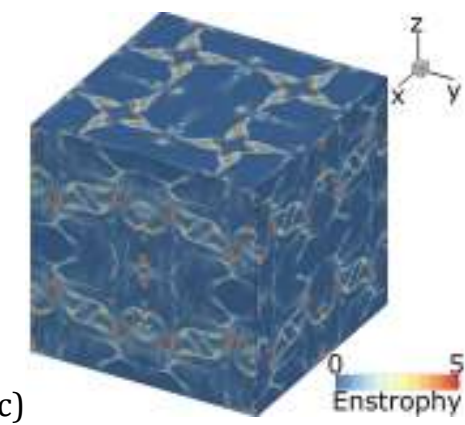

Figure 16: Enstrophy field at $\mathrm{t}=0$ (a), $\mathrm{t}=10(\mathrm{~b})$, and $\mathrm{t}=20$ (c)

(a)
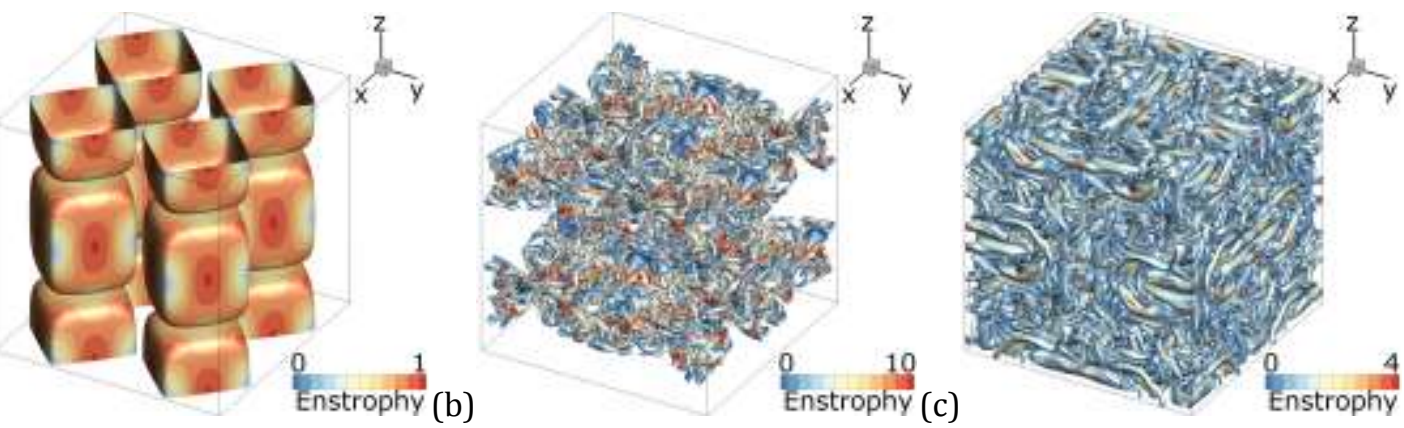

Figure 17: Vortical structures visualised with iso-surfaces of $\lambda_{c i}$ equaling to $1 \%$ of its maximum and coloured with enstrophy. (a): $t=0,(b): t=10,(c): t=20$

\subsection{Turbulent channel Flow}

The performance of the DCOM6 scheme in DNS of wall-bounded flow and its stability in long-time integration are assessed in a turbulent channel flow, in which periodic conditions are applied to the $x$ - and $z$-boundaries, and isothermal no-slip conditions are applied to the walls, as sketched in Figure 19. To drive the flow, an unsteady uniform body force is applied to the $x$-momentum and energy equations in Eq. (30) as,

$$
\begin{gathered}
s_{1}=f_{x}, \\
s_{e}=f_{x} u_{b},
\end{gathered}
$$

in which $f_{x}$ is the body force and $u_{b}$ is the bulk velocity. The body force is calculated based on its previous value, the wall friction and the mass flux at the current step to ensure a constant mass flux, following the procedure given by Lenormand et al. [75]. The size of the domain is $L_{x} \times L_{y} \times L_{z}=[0,2 \pi] \times[0,2] \times[0,4 / 3 \pi]$, matching the setting of Moser et al. [76] 


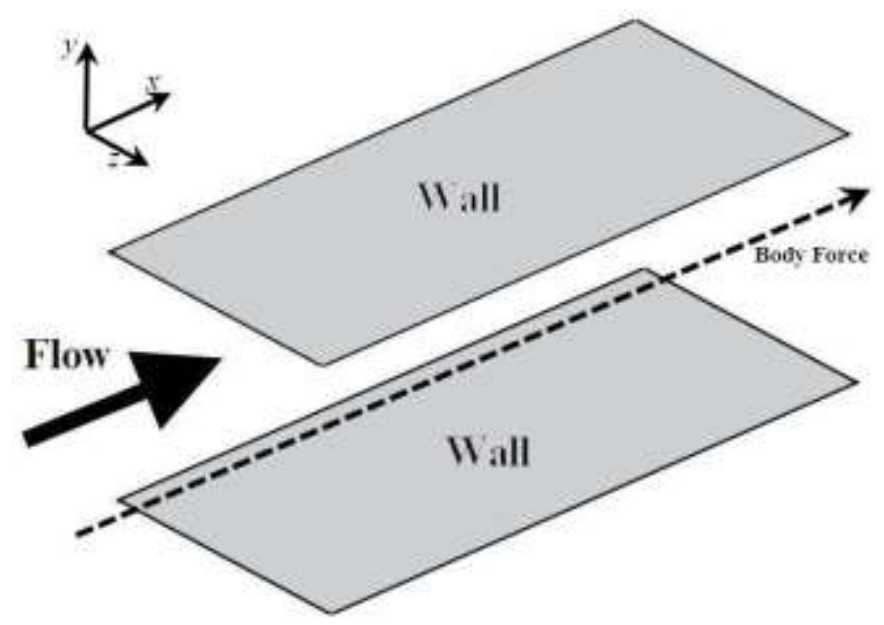

Figure 18: Sketch of open turbulent channel flow

The initial condition is a laminar profile with superimposed random fluctuations up to $30 \%$ of the mean velocity,

$$
\begin{gathered}
u=1.5\left(1-(y-1)^{2}\right)(1+0.3 \epsilon), \\
v=w=0, \\
T=1+\frac{\gamma-1}{3} \operatorname{Pr} \cdot M^{2} 1.5\left(1-(y-1)^{4}\right),
\end{gathered}
$$

where $\epsilon$ is a random number within the range $[-1,1]$.

At the mesh node on the wall and the first node away from the wall, the stencil of the scheme has to be shortened to fit the boundary. Therefore, $2^{\text {nd }}$ and $4^{\text {th }}$-order boundary schemes are used at the wall and at the first node away from the wall, respectively $[7,8,48]$,

$$
\begin{gathered}
\tilde{\phi}_{0}^{\prime}+\tilde{\phi}_{1}^{\prime}=\frac{2}{\Delta}\left(\phi_{1}-\phi_{0}\right), \\
\frac{1}{4} \tilde{\phi}_{0}^{\prime}+\tilde{\phi}_{1}^{\prime}+\frac{1}{4} \tilde{\phi}_{2}^{\prime}=\frac{3}{4 \Delta}\left(\phi_{2}-\phi_{0}\right),
\end{gathered}
$$

for the first derivative terms and

$$
\begin{gathered}
\tilde{\phi}_{0}^{\prime \prime}=\frac{1}{\Delta^{2}}\left(2 \phi_{0}-5 \phi_{1}+4 \phi_{2}-\phi_{3}\right), \\
\frac{1}{10} \tilde{\phi}_{0}^{\prime \prime}+\tilde{\phi}_{1}^{\prime \prime}+\frac{1}{10} \widetilde{\phi}_{2}^{\prime \prime}=\frac{6}{5 \Delta^{2}}\left(\phi_{2}-2 \phi_{1}+\phi_{0}\right),
\end{gathered}
$$

for the $2^{\text {nd }}$ derivative terms.

The Reynolds number based on the bulk velocity, bulk density, the half-height of the channel and the viscosity at the wall is $R e=2,897.5$, corresponding to a friction Reynolds number $R e_{\tau}=183.2$. The Mach number based on the bulk velocity and the speed of sound at the wall is $M a=0.5$. A mesh with $6,291,456(256 \times 192 \times 128)$ nodes is used to discretise the domain. These nodes are evenly distributed in the $x$ - and $z$-directions, and concentrated towards the walls in the $y$-direction. The detailed information of the mesh 
resolution is presented in Table 1, in which the superscript ' + ' stands for variables in wall units.

Table 1. Domain and mesh information of the DNS of turbulent channel flow

\begin{tabular}{llllll}
\hline$L_{x}$ & $L_{y}$ & $L_{z}$ & $N_{x}$ & $N_{y}$ & $N_{z}$ \\
\hline $4 \pi$ & 2 & $4 / 3 \pi$ & 256 & 192 & 128 \\
\hline$\Delta x$ & $\Delta y_{1} \sim \Delta y_{m}{ }^{*}$ & $\Delta z$ & $\Delta x^{+}$ & $\Delta y_{1}^{+} \sim \Delta y_{m}^{+}$ & $\Delta z^{+}$ \\
\hline 0.0491 & $0.00326 \sim 0.0171$ & 0.0327 & 8.99 & $0.598 \sim 3.128$ & 5.99 \\
\hline
\end{tabular}

${ }^{*} \Delta y_{1}$ and $\Delta y_{m}$ are the mesh size of the first node away from the wall and the node in the middle of the channel.

The simulation is run in parallel using 768 processors and the domain is split into 16,8 and 6 subdomains in the $x^{-}, y$ - and $z$-directions, respectively. The non-dimensional time step of the simulation is $1.25 \times 10^{-3}$. The temporal evolution of spatially averaged skin friction is shown in Figure 19, from which we can see the flow goes through a laminar-turbulent transition in the early stage of the simulation and reaches a statistically steady state around $t=100$. For further discussions on the division of stages in late transition, see Ref. [77]. The data collection begins at $\mathrm{t}=193.8$, and 3400 samples are used to calculate the statistics.

The instantaneous turbulent structures in the fully developed channel are illustrated in Figure 20. The classic hairpin counter-rotating vortices [72] and streamwise elongated lowspeed streaks are well captured.

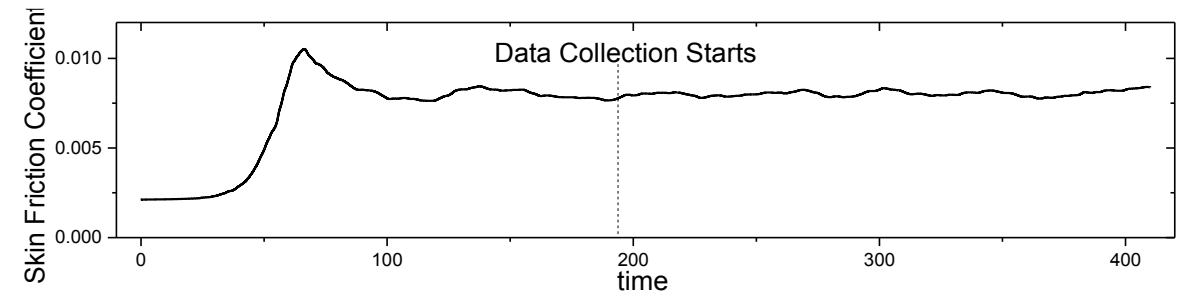

Figure 19: Temporal profiles of spatially averaged skin friction coefficient.

(a)

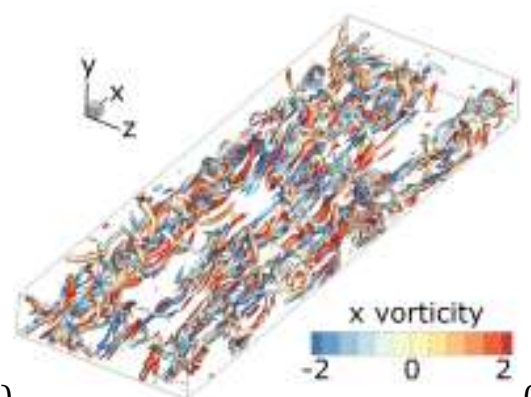

Figure 20: Instantaneous turbulent coherent structures in the lower half of the channel visualised with iso-surfaces of $\lambda_{\boldsymbol{c}}$ equaling to $1.25 \%$ of its maximum and colored with streamwise vorticity (a) and velocity streaks at $\mathrm{y}^{+}=10(\mathrm{~b})$.

The mean velocity profile, root-mean square (RMS) velocity fluctuations and Reynolds shear stress in wall units are presented in Figure 21. The incompressible DNS data of 
channel flow at a similar Reynolds number obtained by Moser et al. [76] are plotted as a reference. Good agreement between the present DNS using the DCOM6 scheme and the incompressible DNS data of Moser et al. [76] is achieved. Therefore, the applicability of the proposed DCOM6 scheme for DNS of wall-bounded turbulence and long-time integration is validated.
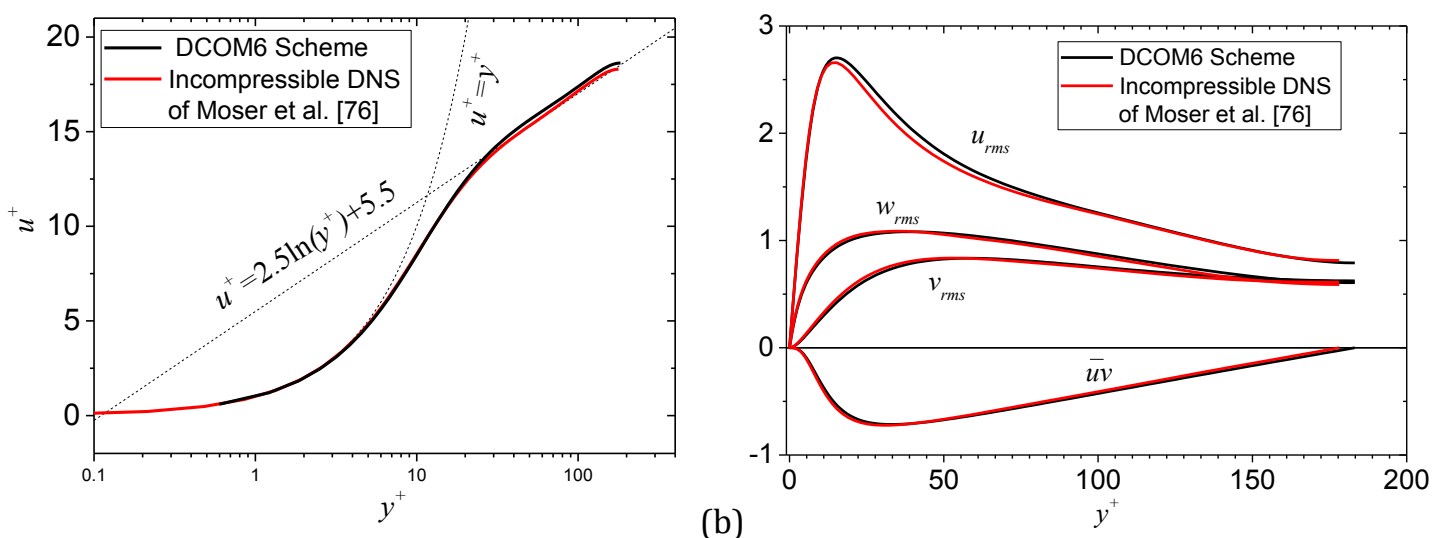

(a)

(b)

Figure 21: (a): Mean velocity profile and (b): RMS velocity fluctuations and Reynolds shear stress. All variables are normalised with friction velocity, density and viscosity at the wall

\subsection{Scalability}

The scalability of the DCOM6 schemes in the TGV problem with a $512^{3}$ mesh is evaluated using ASTR on the HAZEL HEN machine [78], with up to 12288 cores. The wall time consumption per time step (this is obtained by averaging the wall time consumed for 100 steps) with different numbers of processors are listed in Table 2, and the data are plotted in Figure 22. A clear linear acceleration with a 0.9 slope is obtained, indicating that the proposed schemes are well adapted to domain-decomposition-based parallel computation.

Table 2. Scalability of the DCOM6 scheme based on ASTR code and HAZEL HEN machine

\begin{tabular}{lllll}
\hline HPC Nodes & Cores & Time per step $(s)$ & Speedup & Ideal Speedup \\
\hline 4 & 96 & 25.63 & 1.0 & 1.00 \\
\hline 8 & 192 & 12.94 & 1.98 & 2.00 \\
\hline 16 & 384 & 6.62 & 3.87 & 4.00 \\
\hline 32 & 768 & 3.54 & 7.24 & 8.00 \\
\hline 64 & 1536 & 1.86 & 13.81 & 16.00 \\
\hline 128 & 3072 & 0.96 & 26.78 & 32.00 \\
\hline 256 & 6144 & 0.53 & 48.44 & 64.00 \\
\hline 512 & 12288 & 0.29 & 87.65 & 128.00 \\
\hline
\end{tabular}




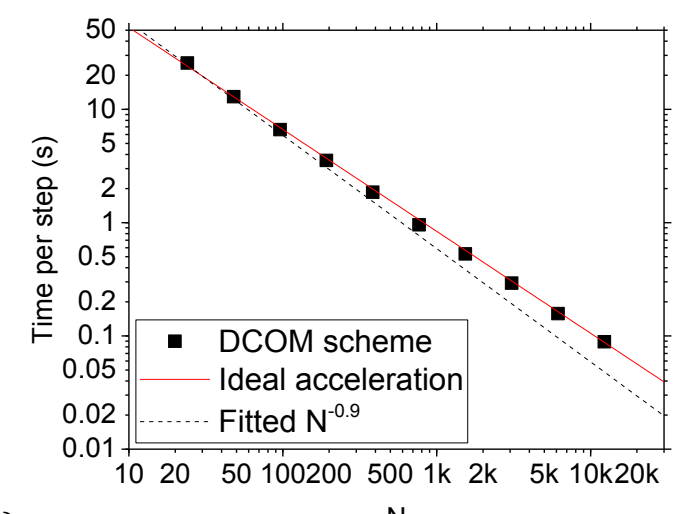

(a)

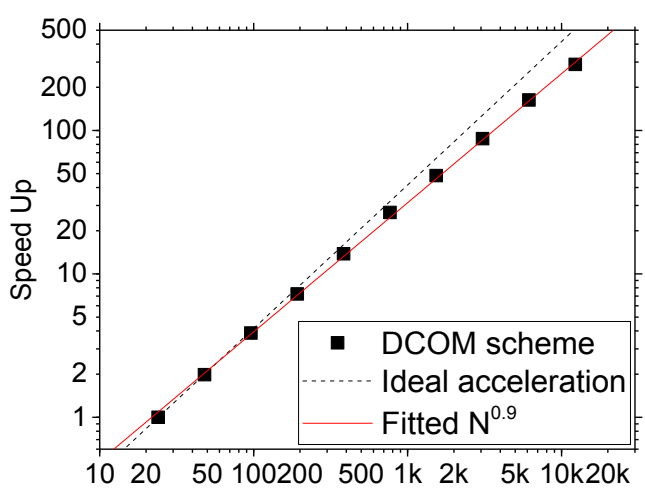

(b)

Figure 22: CPU time per time step (a) and speedup (b)

\section{Summary and Conclusions}

A class of domain-decoupled compact schemes for $1^{\text {st }}$ and $2^{\text {nd }}$ derivatives is proposed for domain-decomposition-based parallel computational fluid dynamics. The compact scheme is decoupled at the interdomain interface, and the tridiagonal matrix system is closed using an explicit scheme of the same order on halo nodes. The accuracy of the scheme can be further improved with the implementation of a correction step with an increased cost of computing resources. The proposed scheme can also be applied for multi-block structured solvers using compact schemes.

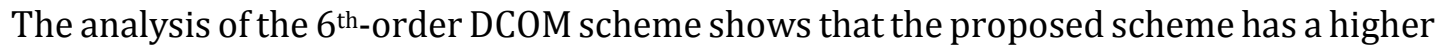
spectral resolution, smaller truncation error, and produces weaker wiggles than Chao's scheme of the same order and the non-dissipative property of a central scheme is well preserved. A correction step can further improve the accuracy of the DCOM scheme in all aspects and restore the scheme towards results obtained by a fully-coupled compact scheme, although it requires additional computational time. The DCOM6 scheme was tested for the 1-D wave-packet propagation and 2-D vortex convection problems, showing improved performance against Chao's scheme in terms of accuracy and independence on the degree of parallelism. The application of the DCOM6 scheme without any correction step in DNS of the Taylor-Green vortex problem and turbulent channel flow demonstrates its suitability in domain-decomposition-based parallel solution for DNS of turbulent flows. In addition, the scalability of the proposed scheme is evaluated on the HAZEL HEN machine with up to 12288 cores, showing that a linear speed-up can be achieved.

\section{Acknowledgement}

The project is supported by the Engineering and Physical Sciences Research Council (EPSRC) through the UK Consortium on Turbulent Reacting Flows (Grant numbers EP/K024574/1 and EP/R029369/1) and the UK Turbulence Consortium (Grant numbers EP/L000261/1 
and EP/R029326/1). D.R.E. gratefully acknowledges support from EPSRC under grants EP/N016602/1, EP/N033841/1 and P022243/1. We would like to thank PRACE for awarding us access to HAZEL HEN at GCS@HLRS, Germany through their Preparatory Access Type B and EPSRC for the use of ARCHER.

\section{References}

[1] Z. Kopal, Numerical analysis, with emphasis on the application of numerical techniques to problems of infinitesimal calculus in single variable, Wiley, New York; Chapman \& Hall, London, 1955.

[2] H. Kreiss, and J. Oliger, Comparison of accurate methods for the integration of hyperbolic equations. Tellus, 1972, 24, 199-215.

[3] H. Kreiss, and J. Oliger, Methods for the Approximate Solution of Time Dependent Problems. GARP Publication Series No. 10, World Meteorological Organization, 1973.

[4] Y. Adam, A Hermitian finite difference method for the solution of parabolic equations. Computers \& Mathematics with Applications, 1975, 1(3-4), 393-406.

[5] Y. Adam, Highly accurate compact implicit methods and boundary conditions. Journal of Computational Physics, 1977, 24(1), 10-22.

[6] R.S. Hirsh, Higher order accurate difference solutions of fluid mechanics problems by a compact differencing technique. Journal of Computational Physics, 1975, 19(1): 90-109.

[7] S. K. Lele. Compact finite difference schemes with spectral-like resolution, Journal of Computational Physics, 1992, 103:16-42.

[8] M. R. Visbal, and D. V. Gaitonde, On the use of higher-order finite-difference schemes on curvilinear and deforming meshes, Journal of Computational Physics, 2002, 181, 155-185.

[9] S. Laizet, and E. Lamballais. High-order compact schemes for incompressible flows: A simple and efficient method with quasi-spectral accuracy, Journal of Computational Physics, 2009, 228: 5989-6015.

[10] S. Pirozzoli, M. Bernardini, and F. Grasso, Characterization of coherent vortical structures in a supersonic turbulent boundary layer, Journal of Fluid Mechanics, 2008, 613: 205-231.

[11] S. Bhaumik and T. K. Sengupta, Precursor of transition to turbulence: Spatiotemporal wave front, Physical Review E, 2014, 89, 043018.

[12] S. Pushpender, T.K. Sengupta, and S. Bhaumik, Three-dimensional transition of zero-pressuregradient boundary layer by impulsively and nonimpulsively started harmonic wall excitation. Physical Review E, 2018, 98, 053106.

[13] M. R. Visbal, and D. P. Rizzetta, Large-eddy simulation on curvilinear grids using compact differencing and filtering schemes, Journal of Fluids Engineering, 2002, 124: 836-847.

[14]S. Nagarajan, S. K. Lele, and J. H. Ferziger, A robust high-order compact method for large eddy simulation, Journal of Computational Physics, 2003, 191(2): 392-419.

[15] Gamet, L., F. Ducros, F. Nicoud, and T. Poinsot, Compact finite difference schemes on nonuniform meshes. Application to direct numerical simulations of compressible flows, International Journal for Numerical Methods in Fluids, 1999, 29:159-191.

[16] E. K. Koutsavdis, G. A. Blaisdell, and A. S. Lyrintzis, Compact schemes with spatial filtering in computational aeroacoustics, AIAA Journal, 2000, 38(4), 713-715.

[17] A. Povitsky, and P.J. Morris, A higher-order compact method in space and time based on parallel implementation of the Thomas algorithm, Journal of Computational Physics, 2000, 161:182-203.

[18] R.D. Sandberg, Direct numerical simulations for flow and noise studies, Procedia Engineering, 2013,61: $356-362$.

[19] N. H. Naik, V. K. Naik, and M. Nicoules, Parallelization of a class of implicit finite difference schemes in computational fluid dynamics, International Journal of High Speed Computing, 1993, 5, 1-50.

[20] X. Guo, and L. Zhang, Symmetric Super compact difference scheme of the Navier-Stokes Equation and its parallel algorithm, Chinese Journal of Computational Physics, 2006, 5(23): 281-289.

[21] C. Ho and S. Johnsson, Optimizing tridiagonal solvers for on boolean cube multiprocessors, SIAM Journal on Scientific Computing, 1990, 11, 563-592. 
[22] X. H. Sun, Application and accuracy of the parallel diagonal dominant algorithm, Parallel Computing, 1995, 21: 1241-1267.

[23] S. Abide, M. S. Binous, and B. Zeghmati, An efficient parallel high-order compact scheme for the 3D incompressible Navier-Stokes equations, International Journal of Computational Fluid Dynamics, 2017, 31(4-5): 214-229.

[24] J. Hofhaus, and E. F. Van de Velde, Alternating-direction line-relaxation methods on Multicomputers, SIAM Journal on Scientific Computing, 1996, 17(2), 454-478.

[25] A. Quarteroni, and A. Valli, Domain decomposition methods for partial differential equations, Oxford University Press, Oxford, UK, 1999.

[26] T.K. Sengupta, A. Dipankar, and A. K. Rao, A new compact scheme for parallel computing using domain decomposition, Journal of Computational Physics, 2007, 220: 654-677.

[27] J. Chao, A. Haselbacher, and S. Balachandar, A massively parallel multi-block hybrid compactWENO scheme for compressible flows, Journal of Computational Physics, 2009, 228: 7473-7491.

[28] J. W. Kim, and R. D. Sandberg, Efficient parallel computing with a compact finite difference scheme, Computers \& Fluids, 2012, 58: 70-87.

[29] J. W. Kim, Quasi-disjoint pentadiagonal matrix systems for the parallelization of compact finitedifference schemes and filters, Journal of Computational Physics, 2013, 241: 168-194.

[30] S. Pirozzoli, Conservative Hybrid compact-WENO schemes for shock-turbulence interaction. Journal of Computational Physics, Volume, 2002. 178(1), 81-117.

[31] Y.X. Ren, M. Liu, and H. Zhang, A characteristic-wise hybrid compact-WENO scheme for solving hyperbolic conservation laws, Journal of Computational Physics, 2003. 192(2), 365-386.

[32] V.F. Fico, D. R. Emerson, and J. M. Reese. A parallel compact-TVD method for compressible fluid dynamics employing shared and distributed-memory paradigms, Computers \& Fluids, 2011, 45: 172-176.

[33] M. H. Carpenter, J. Nordström, and D. Gottlieb, A stable and conservative interface treatment of arbitrary spatial accuracy, NASA/CR-1998-206921, ICASE Report No. 98-12, Langley Research Center, Hampton, VA, 1998.

[34] J. Nordström, and M. H. Carpenter, Boundary and interface conditions for high-order finitedifference methods applied to the Euler and Navier-Stokes equations, Journal of Computational Physics, 1999, 148, 621-645.

[35] J. Nordström, J. Gong, E. van der Weide, M. Svärd, A stable and conservative high order multiblock method for the compressible Navier-Stokes equations, Journal of Computational Physics, 2009, 228, 9020-9035.

[36] F. Olsson, and N. A. Petersson, Stability of interpolation on overlapping grids, Computers and Fluids, 1996, 25, 583-605.

[37] J. Gao, A block interface flux reconstruction method for numerical simulation with high-order finite difference scheme, Journal of Computational Physics, 2013, 241, 1-17.

[38] J.W. Kim, Optimised boundary compact finite difference schemes for computational aeroacoustics. Journal of Computational Physics, 2007, 225, 995-1019.

[39] S. Narayanan, P. Chaitanya, S. Haeri, P. Joseph, J. W. Kim, and C. Polacsek, Airfoil noise reductions through leading edge serrations, Physics of Fluids, 2015, 27, 025109.

[40] J.W. Kim, S. Haeri, and P. F. Joseph, On the reduction of aerofoil-turbulence interaction noise associated with wavy leading edges, Journal of Fluid Mechanics, 2016, 792, 526-552.

[41] J. M. Turner, and J.W. Kim, Aeroacoustic source mechanisms of a wavy leading edge undergoing vortical disturbances. Journal of Fluid Mechanics, 2017, 811, 582-611.

[42] M. Keller, and M. Kloker, DNS of Effusion Cooling in a Supersonic Boundary-Layer Flow: Influence of Turbulence. 44th AIAA Thermophysics Conference, Fluid Dynamics and Co-located Conferences, 2013, AIAA Paper 2013-2897.

[43] M. Keller, and M. Kloker, Direct Numerical Simulations of Film Cooling in a Supersonic Boundary-Layer Flow on Massively-Parallel Supercomputers," Sustained Simulation Performance 2013, edited by Resch, M., Bez, W., Focht, E., Kobaysahi, H., and Kovalenko, Y., Springer, Heidelberg, 2013, 107-128.

[44] M. Keller, and M. Kloker, Effusion Cooling and Flow Tripping in Laminar Supersonic BoundaryLayer Flow. AIAA Journal, 2015, 53(4) 902-919.

[45] M. Keller, and M. Kloker, Direct Numerical Simulation of Foreign-Gas Film Cooling in Supersonic Boundary-Layer Flow. AIAA Journal, 2017, 55(1), 99-111. 
[46] C. Wenzel, B. Selent, M. Kloker, and U. Rist, DNS of compressible turbulent boundary layers and assessment of data/scaling-law quality. Journal of Fluid Mechanics, 2018, 842(10), 428-46.

[47] F. Capuano, A. Mastellone, and E.M. De Angelis, A conservative overlap method for multi-block parallelization of compact finite-volume schemes, Computers and Fluids, 2017, 159, 327-337.

[48]D.V. Gaitonde, and M. R. Visbal, High order schemes for Navier-Stokes equations algorithm and implementation in FDL3DI, 1998, Air Force Research Laboratory, AFRL-VA-WP-TR-1998-3060.

[49] M.H. Carpenter, D. Gottlieb, and S. Abarbanel, Time-Stable Boundary Conditions for FiniteDifference Schemes Solving Hyperbolic Systems Methodology and Application to High-Order Compact Schemes. Journal of Computational Physics, 1994. 111(2): 220-236.

[50] T.K. Sengupta, G. Ganeriwal, and S. De, Analysis of central and upwind compact schemes. Journal of Computational Physics, 2003. 192(2): 677-694.

[51] J. Larsson, S.K. Lele, and P. Moin, Effect of numerical dissipation on the predicted spectra for compressible turbulence, Technical Report Annual Researcg Briefs, Center for Turbulence Research, Stanford University, 2007.

[52] E. Johnsen, J. Larsson, A. V. Bhagatwala, W. H. Cabot, P. Moin, B. J. Olson, P. S. Rawat, S. K Shankar, S. Bjorn, H.C. Yee, X. Zhong, and S.K. Lele, Assessment of high-resolution methods for numerical simulations of compressible turbulence with shock waves, Journal of Computational Physics, 2010, 229, 1213-1237.

[53] J. Fang, Z. Li, and L. Lu, An optimized low-dissipation monotonicity-preserving scheme for numerical simulations of high-speed turbulent flows, Journal of Scientific Computing, 2013, 56, 67-95.

[54]J. Fang, Y. Yao, Z. Li, and L. Lu, "Investigation of low-dissipation monotonicity-preserving scheme for direct numerical simulation of compressible turbulent flows," Computer \& Fluids, 2014, 104, $55-72$.

[55] C. Bogey, and C. Bailly, A family of low dispersive and low dissipative explicit schemes for flow and noise computations, Journal of Computational Physics 194 (2004) 194-214.

[56] A. Dipankar, and T.K. Sengupta, Symmetrized compact scheme for receptivity study of 2D transitional channel flow, Journal of Computational Physics, 2006, 215, 245-273.

[57] T.K. Sengupta, Y.G. Bhumkar, M.K. Rajpoot, V.K. Suman, S. Saurabh, Spurious waves in discrete computation of wave phenomena and flow problems. Applied Mathematics and Computation, 2012. 218(18): 9035-9065.

[58] C. A. Kennedy, and A. Gruber, Reduced aliasing formulations of the convective terms within the Navier-Stokes equations for a compressible fluid, Journal of Computational Physics, 2008, 227: 1676-1700.

[59] A. G. Kravchenko, and P. Moin, On the effect of numerical errors in large eddy simulations of turbulent flows, Journal of Computational Physics, 1997, 131: 310-322.

[60] S. Pirozzoli, Numerical methods for high-speed flows, Annual Review of Fluid Mechanics, 2011, 43, 163-194.

[61] S. Pirozzoli, and M. Bernardini, Turbulence in supersonic boundary layers at moderate Reynolds number, Journal of Fluid Mechanics, 2011, 688, 120-168.

[62] F. Ducros, F. Laporte, T. Souleres, V. Guinot, P. Moinat, and B. Caruelle, High-order fluxes for conservative skew-symmetric-like schemes in structured meshes: Application to Compressible Flows, Journal of Computational Physics, 2000, 16, 114-139.

[63] S. Gottlieb, and C.-W. Shu, Total variation diminishing Runge-Kutta schemes, Mathematics of Computation, 1998, 67(221): 73-85.

[64] D. Gottlieb, and E. Turkel, Dissipative two-four methods for time dependent problems, Mathematics of Computation, 1976, 30, 703.

[65] F. Davoudzadeh, H. McDonald, and B. E. Thompson, Accuracy evaluation of unsteady CFD numerical schemes by vortex preservation, Computer and Fluids, 1995, 24, 883.

[66] H. C. Yee, N. D. Sandham, and M. J. Djomehri, Low-dissipative high-order shock-capturing methods using characteristic-based filters. Journal of Computational Physics, 1999, 150, 199-238.

[67] N. D. Sandham, Q. Li, and H. C. Yee, Entropy splitting for high-order numerical simulation of compressible turbulence, Journal of Computational Physics, 2002, 178: 307-322.

[68] G. I. Taylor, and A. E. Green, Mechanism of the production of small eddies from large ones, Proceedings of the Royal Society A, 1937, 158(895): 499-521.

[69] D. Drikakis, C. Fureby, F.F. Grinstein, and D. Youngs, Simulation of transition and turbulence decay in the Taylor-Green vortex, Journal of Turbulence, 2007, 8 (20). 
[70] C. W. Shu, W. S. Don, D. Gottlieb, O. Schilling, and L. Jameson, Numerical convergence study of nearly incompressible, inviscid Taylor-Green vortex flow, Journal of Scientific Computing, 2005 24 (1), 1-27.

[71] W. M. van Rees, A. Leonard, D. I. Pullin, and P. Koumoutsakos, A comparison of vortex and pseudo-spectral methods for the simulation of periodic vortical flows at high Reynolds numbers, Journal of Computational Physics, 2011, 230(8): 2794-2805.

[72] J. Zhou, R. J. Adrian, S. Balachandar, and T. M. Kendall, Mechanisms for generating coherent packets of hairpin vortices in channel flow, Journal of Fluid Mechanics, 1999, 387: 353-396.

[73] M. J. Ringuette, M. Wu, and M. P. Martin, Coherent structures in direct numerical simulation of turbulent boundary layers at Mach 3, Journal of Fluid Mechanics, 2008. 594, 59-69.

[74] J. Fang, Y. Yao, A. A. Zheltovodov, Z. Li, and L. Lu, Direct numerical simulation of supersonic turbulent flows around a tandem expansion-compression corner, Physics of Fluids, 2015, 27, 125104.

[75] E. Lenormand, P. Sagaut, and L. T. Phuoc, Large eddy simulation of subsonic and supersonic channel flow at moderate Reynolds number, International Journal for Numerical Methods in Fluids, 2000, 32 : 369-406.

[76] R. D. Moser, J. Kim, and N. N. Mansour, Direct numerical simulation of turbulent channel flow up to $\mathrm{Re}_{\tau}=590$, Physics of Fluids, 1999, 11(4): 943-945.

[77] F. Liu, L.P. Lu, and L. Fang, Non-equilibrium turbulent phenomena in transitional channel flows. Journal of Turbulence, 2018, 19(9): 731-753.

[78] https://www.hlrs.de/systems/cray-xc40-hazel-hen/ 